\title{
Altes und Neues zum strafrechtlichen Vorsatzbegriff
}

\author{
Günther Jakobs"
}

A. Vorsatz als Wissen und Wollen?

I. Warum Vorsatz

(oder Fahrlässigkeit)?

II. Wissen als Wollensinhalt............ 285

1. Die Terminologie des Gesetzes. . 285

2. Wollen als Gestalten ............. 286

2. Wollen als Erstreben? ............ 287

3. Ergebnis zu „Wissen als Wollensinhalt"

B. Der Wollensinhalt .................... 291

I. Die Gestaltungsmacht ............. 291

1. Grundlagen - Risikogewöhnung - Ungehorsamsdelikte ........... 291

2. Andere Lösungsvorschläge ...... 294 a. „Abgeschirmte" Gefahr?.... 294

b. „Vorsatzgefahr"?............ 295

3. Ergebnis zu „Gestaltungsmacht" “........................ 297

II. Der Gestaltungsgegenstand des Wollens ................................ 297

1. Der Begriff des Delikts ......... 297

2. Typen von Tatbestandsmerkmalen............................ 299 a. Sogenannte normative Tatbestandsmerkmale

b. Merkmale der objektiven

Zurechenbarkeit............ 301

c. Garantenstellungen........... 302

d. Appellfunktion des Willensinhalts. Zugleich Ergebnis zu „Gestaltungsgegenstand“.... 302

III. Nur präsentes Wissen?............... 304

1. Wertungsfehler versus Wissensfehler.......................... 304

2. Dolus indirectus .............. 306

3. Dolus indirectus und geltendes

Recht. Zugleich Ergebnis zu

„Präsenz des Wissens“.......... 308

IV. Jedes Wissen?....................... 309

1. Sonderwissen ..................... 309

2. Besonderheiten .................. 313

a. Garanten................. 313

b. Eingebrachtes Sonderwis-

sen ...................... 314

3. Ergebnis zu „Jedes Wissen?“.... 315

\section{A. Vorsatz als Wissen und Wollen?}

\section{Warum Vorsatz (oder Fahrlässigkeit)?}

Die Welt der Moderne ist nach einem bekannten Wort von Max Webers „entzaubert", ${ }^{1}$ und das heißt, sie läuft nach Regeln ab, mit denen kein Sinn verbunden ist, die aber gelernt und - im Grundsatz, wenn auch nicht in jedem Einzelfall - zweckmäßig benutzt werden können. Sinn ist nicht vorgegeben, sondern wird durch geschickte Lenkung des Weltverlaufs, handelnd, hergestellt, oder aber er fehlt. Deshalb kann der Täter eines Verbrechens die Verantwortung für sein Verhalten nicht „geheimnisvollen, unberechenbaren Mächten “2 zuschieben (sich etwa als Geißel Gottes oder als Vollstrecker der Natur darstellen), aber auch er kann nicht verantwortlich gemacht werden, wenn er die Welt nicht selbst nach seinem Sinn gestaltet hat, er vielmehr als bloßer Kausalfaktor (oder als noch Kleineres) mit einem Schadensverlauf verbunden ist („Erfolgshaftung“, „Schicksalshaftung “, Haftung eines „Sünden-

* Prof.Dr. Dr. h.c. mult. Günther Jakobs ist emeritierter Professor für Strafrecht, Strafprozessrecht und Rechtsphilosophie an der Rheinischen Friedrich-Wilhelms-Universität in Bonn.

1 M. Weber, Wissenschaft als Beruf, in: Gesammelte Aufsätze zur Wissenschaftslehre (hrsg. von J. Winckelmann), Tübingen 1968, S. 592 (594).

2 Weber, Wissenschaft als Beruf (Fn. 1). - Diesen Mächten wurde (und wird) etwa bei (staatlichen) Verbrechen aus religiösen Gründen die Verantwortung zugeschoben; auch sozialdarwinistisch motivierte Verbrechen gehören hierher („Natur“ als sinnvolle Macht). 
bocks“). Die Befreiung von Verantwortung auf Grund eines „höheren“ Sinns wie die Belastung mit Verantwortung aus einem solchen Grund scheiden beide aus: Verbrechen werden mit einem irdischen Sinn gemacht, oder sie sind keine Verbrechen, vielmehr sinnloser Verlauf der von Zauber freien Welt.

Bei einem Verbrechen handelt es sich um einen Sinnausdruck ${ }^{3}$ des Täters selbst. Ein Sinnausdruck in einer ansonsten sinnlosen Welt ließe sich freilich nicht als Verbrechen darstellen; denn die sinnlose Welt enthält, da sie eben sinnlos ist, weder Verbote noch Gebote. Aber ein Verbrecher ist ja auch nicht „der erste Mensch“4, sondern trifft auf einen Aktionsraum, den Geschichte und Gegenwart bereits mit Sinnansprüchen gefüllt haben: Recht, Religion, Moral, Sitte. Harmoniert das Verhalten des „Täters" mit den rechtlichen Ansprüchen, so ist es nicht verbrecherisch; harmoniert es nicht, so erhält der Sinnausdruck die Eigenschaft eines Sinnwiderspruchs: Neuer Sinn steht gegen etablierten Sinn.

Da sich in einer schlechthin unbekannten Welt kein Sinn ausdrücken und insbesondere keinem Sinn widersprechen lässt, sondern allenfalls um Zufallsergebnisse „gewürfelt" werden kann, muss der Täter eines Verbrechens wissen, wie sein Aktionsraum gestaltet ist, und das heißt zweierlei: Er muss den eingangs genannten Bereich „nackter Tatsachen“ und die Regeln ihrer Nutzung kennen, zudem aber auch die bereits vorhandenen Sinnpräformierungen, also die normative Struktur der Gesellschaft, in der jeder lebt, der eben nicht der erste Mensch ist (und die Zweiteilung „Natur versus Sinn“ ist dabei bereits eine Leistung der Gesellschaft). Beispielhaft, der Täter einer Beleidigung durch moralische Herabsetzung des Opfers muss wissen, dass sich die Rechtslage auf eine bestimmte Moral bezieht, wie eine Beleidigung zu äußern ist, nämlich insbesondere durch sprachliche Symbole, und wie diese durch die Gestaltung der Natur vermittelt werden (Schallwellen, Schriftzeichen).

Alles das ist nicht sonderlich neu, und es wäre auch verwunderlich, wenn im Kernbereich einer so alltäglichen Angelegenheit, wie es die subjektive Seite eines Verbrechens ist, fundamentale Unklarheit herrschen würde. - Das StGB regelt in seinem Allgemeinen Teil die subjektive Seite zwar wenig geglückt (wie noch zu zeigen sein wird; A.II.1.; B.III.3.), aber einigermaßen gründlich ( $\mathbb{\$} \$ 15-21,33,35 \mathrm{StGB})$, dies im Gegensatz zu manchen anderen Verbrechensvoraussetzungen, etwa der (schlecht) so genannten objektiven Zurechnung. ${ }^{5}$ Was das Wissen angeht, so spricht das Gesetz von Kenntnis ( $\mathbb{S} 16$ Abs. 1 Satz 1 StGB) oder Einsicht ( $\$ 17$ Satz 1 StGB), und dieser

3 H. Welzel, Studien zum System des Strafrechts, ZStW 58 (1939), S. 491 (495 ff., 496, 503).

4 Genauer: der erste Sinnproduzent; bei der Annahme eines Schöpfergottes wäre der erste Mensch der zweite Sinnproduzent.

5 Insoweit findet sich nur an einer Stelle eine Andeutung, nämlich zur Einstandspflicht beim Unterlassungsdelikt, $\mathbb{} 13$ Abs. 1 StGB; siehe G. Jakobs, Die strafrechtliche Zurechnung von Tun und Unterlassen, Paderborn 1996, S. 36 ff. und passim. 
Terminologie folgt auch der anschließende Text, ohne dass damit eine begriffliche Differenz von Wissen, Kenntnis und Einsicht behauptet würde. ${ }^{6}$

\section{Wissen als Wollensinhalt}

\section{Die Terminologie des Gesetzes}

Kenntnis allein führt nicht zu einer Gestaltung der Welt; sie mag sich vielmehr in der mehr oder weniger großen Gewissheit des ohnehin Bleibenden und des sich Verändernden erschöpfen. (1) Die übliche Lehre spricht deshalb beim Vorsatz von einem „Wissen und Wollen“ der Tatbestandsverwirklichung ${ }^{7}$ und (2) das Gesetz von der Kenntnis oder Einsicht „,bei Begehung der Tat“ (\$ 16 Abs. 1 Satz 1 und 2 StGB, $\$ 17$ Satz 1 StGB). Beides kann wenig befriedigen: (1) Die Formel vom Wissen und Wollen der Tatbestandsverwirklichung suggeriert eine Trennbarkeit dieser psychischen Vorgänge (dies ganz abgesehen von der verquälten Sprache: Wissen der Tatbestandsverwirklichung?), aber Wissen allein ist ohnmächtig und Wollen allein leer, orientierungslos, jedenfalls also nicht sinnhaft gestaltend. Mit der Formel wird versucht, diesem Ergebnis durch die Verbindung des Wissens mit dem Wollen zu entkommen, aber sie stellt als Addition dar, was Entfaltung eines Begriffs sein sollte: ${ }^{8}$ Ohnmacht plus Leere ist nicht gleich Gestaltung! (2) Und wenn das Gesetz auf Kenntnis oder Einsicht bei der Tatbegehung abstellt, so passt das nicht auf Vorfeld-

6 Insbesondere die Einsicht lässt sich leicht „aufladen“: nicht nur um etwas wissen, sondern es auch verstehen. Aber dieses Verstehen lässt sich auch als bis zum „Grund“ reichendes Wissen oder als entsprechende Kenntnis darstellen. Sehr stark subjektbezogen bei der Einsicht: T. Groteguth, Norm und Verbots(un)kenntnis - $\$ 17$ Satz 2 StGB, Berlin 1993, S. 67 ff./73 ff.; B. Zabel, Aktuelle Begründungs- und Anwendungsprobleme in der Dogmatik zu $\mathbb{1} 17$ StGB, GA 2008, S. 33 (44 ff./53 f.); ders., Schuldtypisierung als Begriffsanalyse. Tiefenstrukturen moderner Praxisformen und deren strafrechtliche Transformation, Berlin 2007, S. $424 \mathrm{ff}$.

7 „Klassisch“ RGSt 51, 305 (311): „Vorsatz bedeutet Wissen und Wollen sämtlicher Tatbestandsmerkmale.“ Weniger additiv („bewußtes Wollen“) RGSt 58, 247 (249); 70, 257 (258). - Nachdrücklich G. Spendel, Zum Begriff des Vorsatzes, in: W. Küper/I. Puppe/J. Tenckhoff (Hrsg.), Festschrift für Karl Lackner, Berlin et al. 1987, S. 187 ff. - Mit Einschränkungen T. Fischer, Strafgesetzbuch, 56. Auflage, München 2009, $\mathbb{1} 15$ Rn. 2 („ungenaue“ Kurzformel); K. Kühl. Strafrecht Allgemeiner Teil, 6. Auflage, München 2008, S. 68 f.; ders. in: K. Lackner/ders., Strafgesetzbuch, 26. Auflage, München 2007, $\mathbb{1 5}$ Rn. 3 („unpräzise“); W. Joecks, in: ders./K. Miebach. (Hrsg.), Münchener Kommentar zum Strafgesetzbuch, Bd. 1, München 2003, $\$ 16$ Rn. 10; C. Roxin, Strafrecht Allgemeiner Teil, Bd. 1, Grundlagen. Der Aufbau der Verbrechenslehre, 4. Auflage, München 2006, $\mathbb{1 2}$ Rn. 4 (aber je nach Vorsatzform in unterschiedlicher Gestalt); P. Cramer/D. Sternberg-Lieben in: Schönke/Schröder, Strafgesetzbuch, 27. Auflage, München 2006, $\$ 15$ Rn. 9 (,unzulängliche“ Beschreibung); H.-J. Rudolphi, in: ders./E. Horn/ E. Samson (Hrsg.), Systematischer Kommentar zum Strafgesetzbuch, 7/8. Auflage, Neuwied Loseblatt, Stand 5/2008), \15 Rn. 1; jeweils mit Nachweisen. Zur Problematik der Formel J. Vogel, in: H. W. Laufhütte/R. Rissing-van Saan/K. Tiedemann (Hrsg.), Strafgesetzbuch. Leipziger Kommentar, Bd. 1, 12. Auflage, Berlin 2007, vor $\$ 15$ Rn. 56 ff. mit Nachweisen.

8 M. Köhler, Strafrecht Allgemeiner Teil, Berlin et al. 1997, S. 149 ff.: „Wissen und Wollen“ verstanden als „die im praktischen Geltungswissen aller Merkmale vollzogene Realisation des objektiven Tatbestandes“ (S. 149); siehe auch W. Kargl, Der strafrechtliche Vorsatz auf der Basis der kognitiven Handlungstheorie, Frankfurt am Main et al. 1993, S. 17 ff./37 ff., zusammenfassend S. 71. 
beteiligte (Tat ist erst die Ausführung): ${ }^{9}$ Die Formulierung des Gesetzes zielt offenbar allein auf selbst Ausführende; ansonsten muss sie auf den Zeitpunkt des zurechenbaren Verhaltens „umgerechnet“ werden. Es kommt noch schlimmer: Unvermeidbare Unkenntnis oder unvermeidbar mangelnde Einsicht bei der Tat kann es nicht geben, da im Fall der Unvermeidbarkeit nichts (vorsätzlich oder fahrlässig) gestaltet, also überhaupt keine Tat im Rechtssinne begangen wird, vielmehr jeglicher rechtliche Sinn ausbleibt und nur Natur waltet. Beispielhaft, wer beim Verlassen seine Büroräume abschließt und nicht weiß oder auch nur wissen kann, dass er damit einen eingedrungenen Dieb einschließt, begeht nicht etwa eine unvermeidbare Freiheitsberaubungstat, sondern insoweit nichts, das man „Tat“ nennen könnte. $\$ 16$ Abs. 1 Satz 1 StGB und $\mathbb{\$} 17$ Satz 1 StGB suggerieren fälschlich, es gebe - im Strafrecht (!) - auch rechtlich gesehen Taten unterhalb von Straftaten.

\section{Wollen als Gestalten}

Zurück zur Formel vom „Wissen und Wollen“! Wissen kann man auch ohne Wollen, wenn das Wollen nicht als völlig nichtssagend („Ich will, dass es Frühling wird.“ „Wollen“ wird zur Metapher für „Wünschen“.), vielmehr als gestaltendes Element verstanden wird: Wer geschehene oder kommende Naturverläufe oder ebensolches Verhalten anderer Personen kennt (ohne, was die kommenden Geschehnisse betrifft, einzugreifen), weiß etwas, ohne auch etwas zu wollen (Erda: „Wie alles war, weiß ich;/ Wie alles wird,/ Wie alles sein wird,/ Seh ich auch." $R$. Wagner, Das Rheingold, Verse $1668 \mathrm{ff}$.). Dieses vom Wollen isolierte Wissen ist möglich, wenn auch ein offenbar für den Vorsatz unbrauchbarer psychischer Befund. Was das Wollen angeht, so ist beim Fehlen jeglichen Wissens jede Orientierung und damit auch eine sinnhafte Gestaltung der Welt ausgeschlossen: Ein solches Wollen würde zum „Zufallsgenerator". Da sich mit einem vom Wollen isolierten Wissen und einem vom Wissen isolierten Wollen strafrechtlich nichts „anfangen“ lässt, müssen Wissen und Wollen zu einem Begriff vereinigt werden: wissendes Wollen oder präsent orientiertes Wollen, was nicht notwendig heißt, das Wollen müsse komplett präsent orientiert sein; bei der Fahrlässigkeit, die hier nicht behandelt wird, fehlt offenbar einiges an präsenter Orientierung, und wie es sich beim dolus indirectus verhält, wird noch darzutun sein (unten II. C.).

Bei diesem Verständnis regeln $\mathbb{1 6}$ Abs. 1 Satz 1 StGB und $\mathbb{1 7}$ Satz 1 StGB Wollensinhalte (genauer: Wollensbezugsinhalte) dergestalt, dass für eine Vorsatztat die eine Tatbestandsverwirklichung gestaltende Wirkung des Wollens gekannt sein muss, und für Unrechtseinsicht eben das Unrecht. Bei einer Vorsatztat ist Wollen im ersten Zugriff - ein Gestalten bei komplett präsentem Orientierungsmaterial; je-

9 Ansonsten bedürfte es keiner Akzessorietät. Es ist freilich ein Irrtum derjenigen Autoren, die für Mittäterschaft ein Mitwirken bei der Ausführung verlangen (Roxin, Strafrecht AT (Fn. 7), Bd. 2: Besondere Erscheinungsformen der Straftat, München 2003, \$2 2 Rn. 198 ff./203, mit Nachweisen), Zurechnung per Akzessorietät lasse sich vermeiden: Nur der zuletzt Handelnde verursacht unmittelbar. 
der Umstand, „der zum gesetzlichen Tatbestand gehört“ (\$16 Abs. 1 Satz 1 StGB), muss als Wollensinhalt präsent sein. So verstanden - Wollen bei der Vorsatztat als orientiertes Wollen - macht es keinen Unterschied, ob die Orientierung eine sogenannte Hauptfolge (eine angestrebte Folge) oder eine Nebenfolge (eine mit dem Angestrebten objektiv zurechenbar verbundene, ihrerseits aber nicht angestrebte Folge) betrifft: Bei kompletter Präsenz des Orientierungsmaterials verläuft die Orientierung bei Haupt- und Nebenfolgen völlig gleich: Der Wille ist orientiert.

\section{Wollen als Erstreben?}

Diesem Verständnis entgegengesetzt arbeitet die weit überwiegende Lehre mit einem nicht funktional, sondern psychologisierend gebildeten Begriff des Wollens, wobei „Wollen“ mit einem „Erstreben“, „Darauf-aus-Sein“, zumindest aber „Billigen“ gleichgesetzt wird. ${ }^{10}$ Dieser Willensbegriff orientiert sich an der Motivation des Täters - ein Geschehen strebt er an (etwa die Tötung eines Menschen durch das InBrand-Setzen eines Hauses), um ein anderes, das ihm unlieb ist, kommt er dabei nicht herum (etwa um den Tod eines weiteren Menschen, der als Gast des erstgenannten Opfers im Haus weilt). Aber wieso sollte es dem Recht auf diese Innerlichkeit des Täters ankommen?

Der Bundesgerichtshof hat auf diese Frage schon früh eine verräterisch formulierte Antwort gegeben: Die „Billigung des Erfolges“ bedeute „nicht etwa, daß der Erfolg den Wünschen des Täters entsprechen“ müsse; denn „im Rechtssinne“ (!) billige der Täter auch, wenn er „um des erstrebten Zieles willen“ sich damit abfinde, dass der an sich unerwünschte Erfolg eintrete; damit sei der Erfolg für den Fall seines Eintritts gewollt. ${ }^{11}$ Nun ist das Verhältnis von Recht und Psychologie ein weites Feld, aber es sollte doch nicht bezweifelt werden, dass es im Recht immer um Begriffe „im Rechtssinne" geht und dass selbst bei einem - hier nicht einschlägigen - pauschalen Verweis auf einen außerrechtlichen Bereich es das Recht ist, das verweist und den betreffenden Bereich nach seinen Regeln inkorporiert. Zwar kann das Recht nicht nur-normativ bleiben, wenn es in der Gesellschaft, wie sie alltäglich stattfindet, Orientierung bieten soll; denn Rechtspersonen sind über die ihnen zugeschriebenen Leiber an die „natürliche“ Welt gebunden. Aber an welche „natürlichen“ Zustände oder Ereignisse

10 Die Terminologie ist bis hin zum Konfusen uneinheitlich. Teils wird „Erstreben“ mit „Wollen“ gleichgesetzt, so dass bereits mit dem Erstrebten sicher Verbundenes nicht mehr gewollt sein soll: $H$.- $H$. Jescheck/T. Weigend, Lehrbuch des Strafrechts. Allgemeiner Teil, 5. Auflage, Berlin 1996, S. 293, 297 ff.; G. Jakobs, Strafrecht Allgemeiner Teil. Die Grundlagen und die Zurechnungslehre, 2. Auflage, Berlin et al. 1991, Abschn. 8 Rn. 16/18 (diese Terminologie gebe ich auf). - Teils werden die sicher verbundenen Nebenfolgen - nicht aber die ungewiss verbundenen - einem „Wollen“ zugeschlagen: Roxin, Strafrecht AT (Fn. 7), \$12 Rn. 18 f. - Teils wird auch für den dolus eventualis ein Wollen oder ein voluntatives Äquivalent postuliert, so insbesondere in der Rechtsprechung; dazu sogleich im Text und oben Fn. 7. Zur üblichen Begriffsbildung zutreffend kritisch und einen „deskriptiven“ (hier: Erstreben) von einem „normativen“ (hier: Wollen als Rechtsbegriff) Begriff unterscheidend I. Puppe, in: U. Kindhäuser/U. Neumann/H.-U. Paeffgen (Hrsg.), Nomos Kommentar Strafgesetzbuch, Bd. 1, 2. Auflage, Baden-Baden 2005, $\mathbb{1 5}$ Rn. 23 ff.

11 BGHSt 7, 363 (369). 
das Recht anknüpft, entscheidet es selbst und richtet sich nicht nach einer zu anderen Zwecken entwickelten Sachlogik des Verständnisses der Natur, hier insbesondere der Psyche.

Es gibt keinen Grund, aus dem sich das Recht darauf einlassen sollte, die in der Täterpsyche gegebene Aufspaltung des Orientierungsmaterials in Haupt- und Nebenfolgen bei der Bestimmung von Vorsatz und Unrechtseinsicht zu übernehmen. Das „Billigen“, „Wollen im Rechtssinne“, auf das es allein ankommt, ist rechtsfunktional zu bestimmen, also nach dem Maß der Orientierung des Täters, und eine bessere Orientierung als durch die Präsenz aller orientierenden Faktoren ist unmöglich. ${ }^{12}$ Wenn also der Täter den Eintritt irgendeines Ereignisses anstrebt, will er nicht nur dieses, sondern auch alle Folgen, die mit dem Ereignis oder den Akten des Anstrebens verbunden sind, sofern er auch insoweit präsent orientiert ist, mit anderen Worten, der Täter kann nur den gesamten Geschehenskomplex wollen oder überhaupt nicht wollen. Wer also im bereits genannten Beispiel seinen Feind in dessen Haus verbrennen will, will auch dessen von derselben Gefahr bedrohten Gast verbrennen.

Die Lösung unterscheidet sich in ihren Konsequenzen nicht von der sogenannten intellektuellen Theorie zum Vorsatz, ${ }^{13}$ wonach nur das Endziel des Täters und seine Zwischenziele, also die Hauptfolgen, gewollt und die Nebenfolgen lediglich als mit dem Gewollten verbunden präsent sind. Die intellektuelle Theorie kommt auf einem Umweg, nämlich über einen Begriff des Wollens als Erstreben, zum richtigen Ergebnis; aber die Nebenfolgen sind - in rechtlicher Bestimmung - selbst gewollt, und zwar unmittelbar. ${ }^{14}$ Bei dieser Terminologie fällt als Ergebnis geradezu in den Schoß, dass

12 W. Frisch, Vorsatz und Risiko. Grundfragen des tatbestandsmäßigen Verhaltens und des Vorsatzes etc., Köln et al. 1983, S. 255 ff., zusammenfassend S. 494 ff. - Damit ist noch nicht ausgemacht, ob es stets um „Präsenz“ geht und was genau „Präsenz“ heißt; ich verstehe darunter ein gültiges, ernsthaftes Urteil über die gegebene Lage Jakobs, Strafrecht AT (Fn. 10), Abschn. 8 Rn. 23; dazu auch Frisch, a.a.O., S. $218 \mathrm{ff}$.

13 Wissen um die (gewollte) Handlung nebst ihren Folgen: Jakobs, Strafrecht AT (Fn.10), $\int 8$ Rn. 8 mit Nachweisen (diese Terminologie gebe ich auf, wie schon in Fn. 10 ausgeführt wurde); Frisch Vorsatz und Risiko (Fn. 12), S. 255 ff.; U. Kindhäuser, Der Vorsatz als Zurechnungskriterium, ZStW 96 (1984), S. 1 (21 ff./25) u. a. m. Die intellektuelle Theorie wurde insbesondere von E. Schmidhäuser gefördert, zuerst: Vorsatzbegriff und Begriffsjurisprudenz im Strafrecht, Tübingen 1968. - Nach dieser Theorie enthält der Eventualvorsatz das Mindestmaterial für einen Vorsatz und ist in diesem Sinne die Grundform: Frisch Vorsatz und Risiko (Fn. 12), S. 496; Freund, Strafrecht Allgemeiner Teil. Personale Straftatlehre, 2. Auflage, Berlin et al. 2009, $\mathbb{} 7$ Rn. $70 .-$ Siehe auch Fn. 17.

14 Deshalb bedarf es bei Nebenfolgen keines Ersatzes für das nicht gegebene Erstreben des Erfolgs, etwa als seine „Hinnahme“ (G. Küpper, Zum Verhältnis von dolus eventualis, Gefährdungsvorsatz und bewußer Fahrlässigkeit, ZStW 100 (1988), S. 758 ff. (766 f.)) oder Gleichgültigkeit ihm gegenüber (K. Engisch, Untersuchungen über Vorsatz und Fahrlässigkeit, 1930, Nachdruck Aalen 1964, S. 186 ff.). - Auch bringt es keinen Gewinn, auf eine „Entscheidung für die mögliche Rechtsgutsverletzung“ abzustellen (Roxin Strafrecht AT (Fn. 7), $\mathbb{1 2}$ Rn. 23 mit Nachweisen; W. Hassemer, Kennzeichen des Vorsatzes, in: G. Dornseifer u. a. (Hrsg.), Gedächtnisschrift für Armin Kaufmann, Köln et al. 1989, S. 289 (295 f.); J. Bung, Wissen und Wollen im Strafrecht, Frankfurt am Main 2009, S. 166 ff./168): Dass in Kenntnis der Folgen gehandelt wird, ist die Entscheidung. Die Kenntnis muss freilich das 
die Absicht, was die Orientierung betrifft, keine Besonderheiten aufweist, da sie nur ein Unterfall des Wollens ist.

Damit unterscheiden sich Nebenfolgen im allein maßgeblichen Bereich der präsenten Orientierung in nichts von den Hauptfolgen; denn auch die Hauptfolgen gehören nicht deshalb zum Vorsatz, weil der Täter sie erstrebt, sondern weil ihr Eintritt als Inhalt des Wollens und damit als orientierender Umstand präsent ist. ${ }^{15}$ Es geht beim Vorsatz nicht um ein Erstreben, sondern um den Gebrauch von Gestaltungsmacht; Beigaben (wie ein „Erstreben“) sind irrelevant; ${ }^{16}$ bleiben sie aus, so ist das unschädlich. Der Umfang der erkannten Gestaltung ist genuin Inhalt des Wollens und bestimmt in dieser Eigenschaft, ohne emotiv gefärbte Ergänzungen, den Umfang des Vorsatzes.

Es gilt also, sich von dem Gedanken zu lösen, der Täter könne aus dem von ihm bei gegebener Orientierung! - gestalteten Komplex einige Teile (Nebenfolgen) herausschneiden, bei denen die Orientierung problematischer, begründungsbedürftiger wäre als bei dem, was verbleibt (bei den Hauptfolgen). Privatim mag der Täter so verfahren, aber nicht rechtlich, wie ja auch, beispielhaft, ein Kaufmann bei einer Firmenübernahme die Passiva rechtlich nicht problematischer, begründungsbedürftiger übernimmt als die Aktiva. Was zum Vorsatz gehört, entscheidet das Recht gemäß seiner Funktion, nicht aber der Täter durch sein Meinen, Fühlen, Werten oder was auch immer und auch nicht die Psychologie.

Diese Lösung - gewollt ist ein Komplex oder nichts - soll am Fall derjenigen Nebenfolgen verdeutlicht werden, die nicht gewiss eintreten, auf die sich, wenn sie überhaupt präsent sind, also ein dolus eventualis richtet. Kommt es zu einer solchen Folge, so wird die Lage oft so gestaltet sein, dass der Täter, hätte er den Eintritt der Folge als gewiss erkannt, seine Handlung nicht vollzogen hätte, weil der fällig werdende „Preis“, eben der dem Täter unliebsame Eintritt der Nebenfolge, den vom Täter erzielbaren Wert übersteigt. Beispielhaft, eine nicht sonderlich wahrscheinlich eintretende, aber auch für den Täter gravierende Nebenfolge (etwa der Tod eines Menschen) ist die Konsequenz des Versuchs, einen eher beiläufigen Vorteil zu erlangen (etwa die Leistung einer Maschine geringfügig zu verbessern). Bei Gewissheit der Nebenfolge hätte mancher Täter wohl schon allein deshalb auf sein Handeln verzichtet, um sich nicht den beim Folgeneintritt unvermeidbaren peinlichen Untersu-

Resultat eines gültigen, ernsthaften Urteils über die Lage sein (Jakobs, Strafrecht AT (Fn. 12), also nicht nur auf schwankenden Vorüberlegungen beruhen; aber das ist bei allen (!) Formen des Vorsatzes der Fall.

15 Sachlich ebenso I. Puppe, Vorsatz und Zurechnung, Heidelberg 1992, S. 37. Zu Puppe auch unten Text zu Fn. 43.

16 So eingehend Frisch, Vorsatz und Risiko (Fn. 12); W. D.. Herzberg, Der Vorsatz als „Schuldform“, als „aliud“ und als „Wissen und Wollen“, in: C. Roxin/G. Widmaier u. a. (Hrsg.), 50 Jahre Bundesgerichtshof. Festgabe der Wissenschaft, Bd. IV, Strafrecht, Strafprozeßrecht, München 2000, S. 51 (78 ff.). 
chungen, Schadensersatzforderungen etc. auszusetzen. Aber um solche Wertungen geht es bei der Bestimmung des Vorsatzumfangs nicht; dieser hängt vielmehr - jenseits aller Wertungen - einzig vom Willensinhalt ab. Der Inhalt besteht - bei unsicherem Folgeneintritt ${ }^{17}$ - aus einer Reihe möglicher Szenarien (mit den Folgen a und b oder mit der Folge a, aber ohne b, etc.), über die der Täter nur als „Paket“ verfügen kann: alle oder keines. ${ }^{18}$ Welches Szenarium aus dem „Paket“ er erstrebt, ist keine Frage des rechtlich bestimmten Wollens; denn es hat mit der Präsenz des Orientierungsmaterials nichts zu tun. Anders formuliert, wenn bekannt ist, was der Täter überblickt, liegt der Inhalt des Wollens fest, und damit lässt sich die Frage nach seinem Vorsatz auch dann sicher entscheiden, wenn nicht ermittelt werden kann, was er erstrebt oder verabscheut und wie schwer Streben und Abscheu für den Täter wiegen.

\section{Ergebnis zu „Wissen als Wollensinhalt“}

Vorsatz ist Wollen der Tatbestandsverwirklichung; Wissen ist der Wollensinhalt. Das Wollen ist von einem psychologisierend bestimmten Erstreben zu unterscheiden: Der Inhalt des Wollens ist das präsente Material zur Orientierung, ohne Unterscheidung von Hauptfolgen und Nebenfolgen, also ohne Blick auf das Bestreben des Täters; denn der Umfang der Orientierung des Täters hängt nicht von seinen Präferenzen ab. - Es handelt sich, nota bene, um einen strafrechtsfunktional gebildeten Begriff des Wollens. In anderen Zusammenhängen mag er enger geschnitten werden, etwa nur Beabsichtigungen umfassen, oder aber auch weiter, sich nämlich auf alle einigermaßen typischen Folgen eines Verhaltens beziehen, so wie bei Molière George Dandin sich selbst die (nicht vorausgesehenen, aber sich aufdrängenden) Folgen seiner (umgekehrten) Mesalliance vorwirft: „Vous l'avez voulu.“ (George Dandin, I, 9). Mehr noch, die Beschränkung des Gewollten auf das präsent Orientierende ist das Ergebnis einer noch vorläufigen Interpretation der $\$ \mathbb{S} 16,17$ StGB; dieses Verständnis wird später durch eine Lehre vom dolus indirectus zu erweitern sein (unten II. C.).

17 Dazu H. Schleehofer, Vorsatz und Tatabweichung, Köln et al. 1996, S. 32 ff./57 ff., zusammenfassend S. 169 (freilich zu einseitig an der Gefahr des „unmittelbaren Ansetzens“ ( $\$ 22 \mathrm{StGB}$ ) orientiert); Herzberg, Vorsatz (Fn. 16), S. 66 ff./82. - Nach hiesigem Verständnis ist nicht nur die Gefahr, sondern auch der Erfolg gewollt, da auch er zum Komplex des orientierenden Materials gehört.

18 Das deckt sich teils mit den Ausführungen von Bung, Wissen und Wollen (Fn. 14), S. 207 f., und seine Kritik an der intellektuellen Theorie (siehe den hiesigen Text zu Fn. 13f.) führt zu einem zumindest terminologisch vorzugswürdigen Ergebnis (deshalb geht meine Kritik in der Buchbesprechung GA 2009, S. 317 (319 f.) zu weit). Aber es gelingt Bung nicht, das Wollen vom Erstreben hinreichend zu lösen und als Rechtsbegriff zu etablieren: Dem Täter sei der Eintritt der Nebenfolge immer noch ,lieber als" ein Verzicht auf die Handlung (S. 208). Das ist, was den Eintritt der Folge angeht, im oben genannten Beispiel offenbar nicht der Fall, ist aber auch irrelevant; denn ein vom Täter verfluchter Erfolg, der präsent orientiert gestaltet wurde, ist gewollt (wenn man so will: im Rechtssinne, siehe oben zu Fn. 11). Die Beziehung des Täters zum Erfolg lässt sich nicht nur „Wollen“ nennen (so Bung, S.271), sondern rechtlich als solches begreifen. Zu Bung siehe L. Greco, Neue Wege der Vorsatzdogmatik, ZIS 13 (2009), S. 813 (818ff.). 


\section{B. Der Wollensinhalt}

\section{Die Gestaltungsmacht}

\section{Grundlagen - Risikogewöhnung - Ungehorsamsdelikte}

Der Inhalt des Wollens beim Vorsatzdelikt ist die - später zu behandelnde - Kenntnis dessen, was gestaltet werden wird, sowie die - hier zur Behandlung anstehende Kenntnis des Umfangs der zur Verfügung stehenden Gestaltungsmacht. Nachdem die ehemals vehement vertretene Ansicht zurückgetreten ist, „allein der Sinn, den der Täter im Tatvorsatz seiner Tat gibt,“ sei „das Wertungssubstrat des Normwidrigkeitsurteils, “ so dass „selbst der abergläubische Versuch ... Unrecht“ sei, ${ }^{19}$ wird überwiegend - eher konstatiert als - argumentiert, Willensinhalt sei bei der Vorsatztat der Gebrauch einer unerlaubten Tatmacht, also die Schaffung eines unerlaubten Risikos, nicht auf eigene Gefahr des Opfers, außerhalb des Vertrauensgrundsatzes wie auch außerhalb des Regressverbots. ${ }^{20}$ Nach dieser Lehre entscheidet nicht der Täter selbst, was sein Verhalten bedeutet, noch weniger ergibt sich das aus einem vom Täter „irgendwie“ angestoßenen Kausalverlauf, vielmehr entscheidet die $G e$ sellschaft, was sie stört; beispielhaft gesprochen, nicht „totbeten “ stört, auch nicht „einen Tod ,irgendwie’ verursachen“, vielmehr stört es, wenn ein Wollen realisiert wird, das nach der gesellschaftlich maßgeblichen Semantik und in diesem Sinn objektiv „töten“ bedeutet, und diese Bedeutung fehlt einem Verhalten, das innerhalb des erlaubten Risikos oder auf eigene Gefahr des Opfers oder im Vertrauen auf die Harmlosigkeit des Verhaltens eines anderen oder im Bereich des Regressverbots vollzogen wird.

Die Berücksichtigung der objektiven Gestalt des unerlaubten Verhaltens ist aber bei der Festlegung des Willensinhalts der Vorsatzdelikte nur der erste Schritt; denn die Annahme, jede minimale Überschreitung der damit bezeichneten Grenze - Kenntnis der Überschreitung sowie der möglichen Folgen vorausgesetzt - reiche zum Vorsatz hin, würde zu geradezu monströsen Ergebnissen führen. Beispielhaft gefragt, wenn ein aktuell optimal kenntnisreicher Autofahrer dort, wo er mit seinem Fahrzeug 50 Stundenkilometer fahren darf, fünf Prozent schneller fährt und jederzeit (aber eben mit äußerst geringer Wahrscheinlichkeit) etwas „passieren“ könnte, was bei regulä-

19 Armin Kaufmann, Zum Stande der Lehre vom personalen Unrecht, in: G. Stratenwerth u. a. (Hrsg.), Festschrift für Hans Welzel, Berlin et al. 1974, S. 393 (403); D. Zielinski, Handlungs- und Erfolgsunwert im Unrechtsbegriff. Untersuchungen zur Struktur von Unrechtsbegründung und Unrechtsausschluß, Berlin 1973, S. 134 mit Fn. 13 und passim. - Diese Lehre, die auf eine Verneinung der objektiven Bestimmung des unerlaubten Verhaltens hinauslaufen soll, kann aus mehreren Gründen nicht durchgehalten werden: (1) Sollte der abergläubische Garant auch Garant zur Vornahme abergläubischer Rettungsunternehmungen sein? (2) Wie hoch soll das noch erlaubte Risiko im Abergläubischen anzusetzen sein? (3) Wieso ist das „Herbeibeten“ der Hilfe Überirdischer mehr als ein Beteiligungsversuch? Zu letzterem, nicht gerade ein Fundamentalproblem, Jakobs, Strafrecht AT (Fn. 10), Abschn. 25 Rn. 22 mit Fn. 33.

20 Zu diesen sich teilweise überschneidenden Instituten der Bestimmung des unerlaubten Verhaltens siehe Jakobs, Strafrecht AT (Fn. 10), Abschn. 7 Rn. 35 ff./51 ff.; $\$ 24$ Rn. 13 ff. 
rem Tempo noch beherrschbar wäre, so liegt bereits ein Versuch der Sachbeschädigung oder Körperverletzung oder gar Tötung vor?

Die genannten Institute zur Regelung der Zurechnung und vice versa der Nicht-Zurechnung sind kein Oktroy des Rechts, sondern werden in der gesellschaftlichen Praxis gewonnen. Dort muss auch die Antwort auf die Frage gesucht werden, weshalb die Vorsatzzurechnung bei minimalen Risikoüberschreitungen völlig überzogen, eben monströs, wirkt. Ein erster - schwacher - Grund dürfte aus der typischen Beweislage folgen: Wann lässt sich schon dartun, dass ein Schadensverlauf gerade auf einer minimalen Risikoüberschreitung beruht und nicht auf dem erlaubten Basisrisiko? Aber es gibt einen weiteren, gewichtigeren Grund, der nunmehr entwickelt werden soll.

Was in der gesellschaftlichen Praxis gewonnen wird, ist in der Regel randunscharf. Beispielhaft zum Regressverbot gefragt, wann genau endet die trennende Arbeitsteilung und beginnt die verbindende - etwa beim Verkauf eines Fleischermessers an einen vielleicht oder gar offenbar zornentbrannten Käufer?21 Der Bereich der Unschärfe kann durch positivrechtliche Regelungen verkleinert werden (etwa durch Straßenverkehrsvorschriften), aber je genauer diese ausfallen, um so mehr steckt in ihnen ein Stück Willkür; denn knapp diesseits oder jenseits der nun einmal festgelegten Linie wäre der Grenzverlauf nicht weniger plausibel. Bei dieser Lage kann der Schnitt zwischen dem Erlaubten und dem Unerlaubten nur in seltenen Fällen anschaulich folgenorientiert verlaufen, dies mit der Konsequenz, dass meist eine leichte Grenzüberschreitung nicht als ernsthaftes ${ }^{22}$ Unternehmen einer Tatbestandsverwirklichung begriffen werden kann. So ist etwa im soeben angeführten Beispiel einer geringfügigen Überschreitung der erlaubten Geschwindigkeit im motorisierten Straßenverkehr das für den Täter wie auch allgemein gültige Urteil ausgeschlossen, für einen nennenswert wahrscheinlichen Unfall sei nunmehr bereits alles getan, was heißt, es liege ein beendeter Versuch vor. ${ }^{23}$ Dabei geht es nicht um eine private Ansicht des Täters; wo dieser die für ihn geltende Grenze zwischen irrelevanter und relevanter Gefahrerhöhung ansetzt, ist rechtlich gleichgültig, wenn der Täter nur das gesellschaftliche Urteil kennt; ${ }^{24}$ für einen gefahrfreudigen Jugendlichen gilt also eben die Grenze, die für jedermann und auch für einen Überängstlichen maßgeblich ist. ${ }^{25}$

21 Dazu G. Jakobs, Beteiligung, in: D. Dölling (Hrsg.), Festschrift für Ernst-Joachim Lampe, Berlin 2003, S. 561 (563 ff.) mit Nachweisen.

22 Wie Fn. 12 a. E.

23 Das bezieht sich nur auf den gesellschaftlichen Alltag. Es mögen nicht-alltägliche Bereiche ausgegrenzt werden, bei denen strengere Grundsätze anzuwenden sind. Etwa beim Umgang mit stark strahlendem Material dürfte weder die Unanschaulichkeit der Grenze ein Argument bilden noch die nachfolgend zu behandelnde Risikogewöhnung.

24 Dazu unten B.II.2.b. - Insoweit wie hier NK-Puppe (Fn. 10), $\mathbb{1} 15$ Rn. 85; kritisch Roxin, Strafrecht AT (Fn. 7), $\$ 12 \mathrm{Rn} .48 \mathrm{ff}$.

25 A. A. insbesondere Köhler, Strafrecht AT (Fn. 8) S. 164 ff.; ders., Die bewußte Fahrlässigkeit. Eine strafrechtlich-rechtsphilosophische Untersuchung, Heidelberg 1982, S. 373 ff. Auf einen „Selbstwi- 
Weiteres kommt hinzu. ${ }^{26}$ Nicht nur für einen - durchaus risikobewussten - Täter bleibt die Gefahr bei minimaler Grenzüberschreitung „im Theoretischen“, vielmehr muss jeder potenzielle Täter alltäglich registrieren, dass er auch Opfer des unerlaubten, wenn auch nur minimal gefährlichen Verhaltens der anderen werden könnte, und wenn er sich an die höchstwahrscheinliche Harmlosigkeit solchen Verhaltens nicht gewöhnt, was heißt, wenn er es nicht ohne Angst um sein Wohl hinnimmt, muss er den betreffenden Lebensbereich, etwa den Straßenverkehr, überhaupt meiden. Beispielhaft, wer auf der Autobahn immer dann, wenn ein Nachfolgender dichter auffährt, als es erlaubt ist, ernsthaft um sein Leben fürchtet, wird sein Fahrzeug wegen seiner permanenten Aufregung gewiss schlechter führen als ein „Hartgesottener".

Das heißt nicht, das Recht solle die Grenze des Erlaubten hinausschieben; sie verläuft sinnvollerweise einigermaßen „früh“; denn ein nur minimal riskantes Verhalten lässt sich zwar isoliert nicht als tatbestandsverwirklichendes Unternehmen begreifen, aber das mag im Blick auf seine tausend- oder gar millionenfache Häufung anders aussehen. Abermals beispielhaft, wenn im Bereich des Straßenverkehrs in Deutschland wöchentlich nur eine Trunkenheitsfahrt eines mäßig alkoholisierten, trinkgewohnten Routiniers in Rede stünde, würde das Verhalten mangels einer nennenswerten Erhöhung des geduldeten Risikos wohl kaum als unerlaubt eingeordnet; allerdings geht es bei massenhaftem Vollzug eines solchen Verhaltens um den sich anschaulich vollziehenden Verlust hochwertiger Güter. Aber es gibt keinen Rückschluss von der anschaulichen Schädlichkeit massenhaften Verhaltens auf die anschauliche Schädlichkeit eines einzelnen Verhaltensvollzugs; denn bei dem Rückschluss geht die Anschaulichkeit verloren, und an die Stelle der Überzeugungskraft der Anschaulichkeit muss dann die Notwendigkeit treten, sich diszipliniert zu verhalten: Das Verletzungsdelikt wandelt sich in ein Ungehorsamsdelikt; ${ }^{27}$ der generelle Verletzungsbezug des Verhaltens ist einsehbar, und damit ist es auch die Notwendigkeit eines Verbots; für den Einzelfall bleibt freilich nur Gehorsam, weil dem Verletzungsbezug insoweit die entscheidungsrelevante Dichte (die Anschaulichkeit) abgeht.

derspruch" des nicht eingebundenen Individuums, also auf den Zustand in einem anderen System als demjenigen des Rechts, könnte es nur ankommen, wenn der Staat als Verwalter des Rechts das Individuelle zu kontrollieren hätte und gegebenenfalls Veränderungen durchsetzen könnte: Das ergäbe freilich einen einigermaßen totalitären Staat. Auf individuelle Ausgestaltungen kann das Recht nur reagieren, wenn diese als „Niederschlag“ externer, etwa gesellschaftlicher Defizite dargestellt werden können, wenn also der Konflikt verlagert werden kann („exotische Sozialisation“; dazu Jakobs, Strafrecht AT (Fn. 10), Abschn. 19 Rn. 7; Timpe, Normatives und Psychisches im Begriff der Vermeidbarkeit eines Verbotsirrtums, GA 1984, S. 51 (54)).

26 Zum folgenden Text: G. Jakobs, Die subjektive Tatseite von Erfolgsdelikten bei Risikogewöhnung, in: W. Frisch u. a. (Hrsg.), Festschrift für Hans-Jürgen Bruns, Köln et al. 1978, S. 31 ff.; ders., Strafrecht AT (Fn. 10), Abschn. 9 Rn. 15.

$27 \mathrm{Zu}$ „Tabupflichten“ eingehend P. Velten, Normkenntnis und Normverständnis, Berlin 2002, S. 237 ff. 


\section{Andere Lösungsvorschläge}

\section{a. „Abgeschirmte“ Gefahr?}

Das Problem minimaler Überschreitung des erlaubten Risikos wird nur - aber immerhin!-vereinzelt erkannt und zutreffend im (realisierten oder, beim Versuch: vorgestellten) objektiven Tatbestand loziert. Was hier zuvor als die praktisch entscheidungsrelevante Dichte der Gefahr einer Tatbestandsverwirklichung bezeichnet wurde (eine objektiv vorliegende oder doch - beim Versuch - vom Täter angenommene qualifizierte Gefahr der tatbestandsverwirklichenden Gestaltung), wird in der Literatur unter den Titeln (1) „nicht abgeschirmte Gefahr“ oder (2) „Vorsatzgefahr“ behandelt.

Die erste (von Herzberg entwickelte) Ansicht setzt bei Phänotypen an: ${ }^{28}$ „Die durch menschliche Aufmerksamkeit (des Täters, des Opfers oder einer dritten Person; ${ }^{29}$ G.J.) abgeschirmte Gefahr“ werde durch eine „hocheffiziente Reservesicherung “ beherrscht, ${ }^{30}$ so dass der Täter von einem „soliden Grund seiner Erfahrung“31 aus urteilen könne, „seine oder fremde Aufmerksamkeit“ würden „die Gefahr aller Voraussicht nach meistern “. ${ }^{32}$ Beispielhaft, der Fahrzeughalter, der seinen bewährten und konkret informierten Chauffeur beauftragt, ein Fahrzeug mit einem leichten Bremsdefekt in die nahe gelegene Werkstatt zu bringen, wird, bei nur minimalen Restzweifeln, von einer „Meisterung“ der Situation durch den Chauffeur ausgehen. ${ }^{33}$

Die skizzierte Lehre überzeugt insoweit, als bei der „Abschirmung“ einer Gefahr diese die aktuell entscheidungsrelevante Dichte verlieren kann; die Lehre dürfte diesen einen Fall zutreffend erfassen, aber eben nur diesen einen: Eine installierte mechanische oder sonst „natürliche“ Anlage kann die Gefahr nicht weniger abschirmen (im Beispiel etwa ein Nachfüllen verlorener Bremsflüssigkeit vor der Fahrt zur Werkstatt). Der eine Fall wird zudem nicht exakt erfasst, ${ }^{34}$ da er nicht einmal umrisshaft eine Antwort auf die Frage gibt, wann die Abschirmung stark genug ist. Fehlt es an hinreichender Stärke, so bleibt ein nicht abgeschirmtes Restrisiko praktisch relevan-

28 R. D. Herzberg, Die Abgrenzung von Vorsatz und bewußter Fahrlässigkeit - ein Problem des objektiven Tatbestands, JuS 1986, S. 249 (254): Es gehe im Ansatz um „ein rein tatsächliches Phaenomen“; kritisch dazu SK-Rudolphi (Fn. 7), $\$ 16$ Rn. 46 a mit Nachweisen.

29 R. D. Herzberg, Das Wollen beim Vorsatzdelikt und dessen Unterscheidung vom bewußt fahrlässigen Verhalten, JZ 1988, S. 573/ 635 (642).

30 Herzberg, Abgrenzung (Fn. 28), S. $254 \mathrm{f}$.

31 Herzberg, Abgrenzung (Fn. 28), S. 254.

32 Herzberg, Abgrenzung (Fn. 28), S. 254; ders., Wollen (Fn. 29), S. 641 f.

33 Was das rollengemäße Bemühen des Chauffeurs angeht, greift der Vertrauensgrundsatz.

34 Herzberg, Abgrenzung (Fn. 28), S. 255: im „Übergangsbereich“ seien „,zweifelsfreie Entscheidungen“ nicht garantiert. - Grenzunschärfe des Ergebnisses ist bei der Beurteilung sozialer Ereignisse ein nur schwaches Gegenargument: Solche Beurteilungen gelingen nie randscharf. 
ter Dichte. ${ }^{35}$ Im Ergebnis geht es also weder nur um Abschirmung durch menschliche Aufmerksamkeit, noch schließt diese stets ein Risiko von vorsatzrelevanter Dichte aus.

Auch unabhängig von den genannten Monita kann an der Lehre nicht befriedigen, dass sie diejenigen Fälle, in denen sich ein trotz der Abschirmung verbleibendes Restrisiko unerlaubter Höhe verwirklicht, wie selbstverständlich der Fahrlässigkeit zuschlägt; ${ }^{36}$ denn wie schon dargelegt wurde, mutiert der verstehbare Norminhalt zumindest in dem Bereich, in dem das Restrisiko alltäglich vielseitig praktiziert, deshalb auch vielseitig erduldet und damit zu etwas Gewöhnlichem wird, vom Verlangen nach Folgenvermeidung zum Verlangen nach Gehorsam. Das aber klammert die Theorie von der abgeschirmten Gefahr aus. Wenn aus einem „soliden Grund der Erfahrung“ 37 die Restgefahr zu vernachlässigen ist, wie sollte sich dann ein Täter wegen dieser Restgefahr zur Vermeidung seines Verhaltens motivieren? ${ }^{38}$ Die oben dargestellte Lehre von der Risikogewöhnung hat demgegenüber den Vorteil, nicht vorsatzspezifisch zu argumentieren: Es geht nicht um die Grenze zwischen Vorsatz und Fahrlässigkeit, sondern zwischen (vorsätzlichen oder fahrlässigen) Erfolgsdelikten und Ungehorsamsdelikten.

\section{b. „Vorsatzgefahr"?}

Die als Zweites zu behandelnde (von Puppe entwickelte) Lehre von der Vorsatzgefahr setzt zutreffend bei der gesellschaftlichen Bedeutung des Täterverhaltens an: „Maßgeblich ist ... nicht, ob der Täter die Gefährdung und Verletzung fremder Integrität tatsächlich gebilligt oder verdrängt hat, sondern ob sein Verhalten, interpretiert als das eines Vernunftwesens, der Ausdruck solcher Billigung ist. " 39 Das soll der Fall sein, wenn zur erforderlichen Größe der Gefahr „,jene anschauliche Typizität des Verletzungsprozesses“ hinzukommt, „die einem nach allgemeinen, gültigen, vernünftigen Maßstäben handelnden Täter nur die Wahl läßt, die Handlung zu unter-

35 I. Puppe, Der Vorstellungsinhalt des dolus eventualis, ZStW 103 (1991), S. 1 (18f.); dies. Vorsatz (Fn. 15), S. 38; B. Schünemann, Vom philologischen zum typologischen Vorsatzbegriff, in: T. Weigend u. a. (Hrsg.), Festschrift für Hans Joachim Hirsch, Berlin et al. 1999, S. 363 (369 f.).

36 Herzberg, Abgrenzung (Fn. 28) und ders., Wollen (Fn. 29), durchgehend.

37 Herzberg, Abgrenzung (Fn. 28), S. 254.

38 Herzberg findet plausible Fälle für Fahrlässigkeit, weil er die relevante Abschirmung zu niedrig ansetzt. Etwa das Zufahren auf einen Polizisten in „Nötigungsabsicht“ (Herzberg, Abgrenzung (Fn. 28), S. 258) wird, wenn es nicht gerade im Schritttempo erfolgt, die Gefahr einer Verletzung allzu dürftig abschirmen: Vorsatz bleibt!

39 Puppe, Vorstellungsinhalt (Fn. 35), S. 14; NK-Puppe (Fn. 10), $\mathbb{1} 15$ Rn. 68 f. - Puppe meint, diese Normativierung der Willensseite, genauer, die Bestimmung des Willens als Rechtsbegriff, könne nicht auf die Wissensseite übertragen werden: „Bis zum Wissen dringen wir im sozialen Kontakt in die Psyche des anderen vor. Eine Normativierung auch des Wissenselements würde sich allzu weit von dem allgemeinen Standard gegenseitiger Beurteilung entfernen “; (Fn. 35), S. 37. Warum aber ein psychologisierend bestimmter Wissensbegriff ein tauglicher Rechtsbegriff sein soll, bleibt offen. 
lassen oder den Erfolg zu akzeptieren. “40 Die damit bezeichnete „Vorsatzgefahr“41 soll nach einer ursprünglichen Konzeption nur für den dolus eventualis maßgeblich sein, während der direkte Vorsatz entweder durch „Wissentlichkeit“ ausgezeichnet sei oder durch den „direkt auf den tatbestandlichen Erfolg“ gehenden Willen (im psychologisierenden Sinn); insoweit bedürfe es keines weiteren „Verbindungsglieds “. ${ }^{42}$ Später wird die Halbheit dieser Lösung erkannt und korrigiert: Auch der Absichtstäter soll nicht etwa deshalb vorsätzlich handeln, weil er etwas erstrebt, sondern weil sein Verhalten „auf einer nach allgemeinen Kriterien sinnvollen Zielverfolgungsstrategie beruht", so dass die Vorsatzgefahr auch zum Vorstellungsinhalt des Absichtstäters gehört. ${ }^{43}$

Was ist eine Vorsatzgefahr? Eine Vorsatzgefahr wird ins Werk gesetzt, wenn vom Täter „eine sinnvolle Strategie der Herbeiführung des Erfolges“ eingeschlagen wird. ${ }^{44}$ Damit scheiden diejenigen „unerlaubte Risiken (Fahrlässigkeitsrisiken)“ aus, „die eine zu geringe Erfolgswahrscheinlichkeit begründen, um eine vernünftige Erfolgsverwirklichungsstrategie darzustellen " 45 Das ist eine problematische Definition. Zwar dürften sich Einwände ausräumen lassen, die sich auf die Relativität zum Arsenal der zur Verfügung stehenden Möglichkeiten beziehen. Beispielhaft, wer seinen Erzfeind sicher vernichten könnte, wählt keine vernünftige Strategie, wenn er so vorgeht, dass die Chance des Gelingens eins zu fünfzig beträgt; wer aber nur über diese kleine Chance verfügt, wählt sie vernünftigerweise. Die Vernünftigkeit darf wohl nicht konkret, sondern muss generell bestimmt werden (Welche Gefahr bildet in irgendeinem Kontext noch eine vernünftige „Erfolgsherbeiführungsstrategie“?). Gewichtiger ist die Frage, woher sich die Bestimmung der „sinnvollen Strategie“ speist. ${ }^{46}$ Der Gesellschaft dürften die Daten kaum vorgegeben worden sein, also sind sie aus ihrer Gestalt herzuleiten, was zuvor (A.I.1.) mit der Berücksichtigung der notwendigen Risikogewöhnung versucht wurde. Isoliert von dieser Verbindung mit der Risikogewöhnung gerät die „sinnvolle Strategie zur Herbeiführung des Erfolges“ deutlich zu eng; denn es geht - entgegen der Lehre von der Vorsatzgefahr - straf-

40 Puppe, Vorstellungsinhalt (Fn. 35), S. 21.

41 Puppe, Vorstellungsinhalt (Fn. 35), S. 39, 40; dies., Vorsatz (Fn. 15), S. 35 ff.

42 Puppe, Vorstellungsinhalt (Fn. 35), S. 39; auch Roxin, Strafrecht AT (Fn. 7), $\mathbb{1} 12$ Rn. 4 hält das Fehlen eines Erstrebens beim dolus eventualis für eine „Schrumpfung der Vorsatzsubstanz“.

43 Puppe, Vorsatz (Fn. 15), S. 37. - Damit erledigt sich wohl auch die frühere Option für die relative (das heißt, von der erreichten Hauptfolge aus berechnete) Bestimmung der Gefahr in Fällen des dolus eventualis.

44 Puppe, Vorsatz (Fn. 15), S. 39. - Puppe hält es für eine „innere Angelegenheit“ des Täters, „wie er sich wirklich damit auseinandersetzt" (S. 39); „wirklich“ meint wohl „psychologisierend bestimmt“ (zutreffend S. 40 f.).

45 Puppe, Vorsatz (Fn. 15), S. 45.

46 Immerhin geht es um eine im Ansatz lebensweltlich bestimmte Wahrscheinlichkeit (zur Kritik der „Wahrscheinlichkeitstheorien“ grundlegend Engisch, Untersuchungen (Fn. 14), S. 209 ff.); das verkennt Schünemann, Vorsatzbegriff (Fn. 35), S. 370 bei seiner Kritik. Aber die Anbindung an die Lebenswelt erfolgt zu punktuell! 
rechtlich nicht um strategisch sinnvolle Erfolgsherbeiführung, sondern um Strategien der Erfolgsvermeidung, und alle Risiken, die außerhalb des Bereichs der notwendigen Gewöhnung liegen, sind wegen des drohenden Erfolges und nicht nur aus Gehorsamsgründen zu vermeiden; es gibt keine reinen „Fahrlässigkeitsrisiken “. ${ }^{47}$ - Nur zu erwähnen bleibt, dass die Lehre von einer „Vorsatzgefahr“ (wie schon diejenige von einer „abgeschirmten Gefahr“) wegen der Fixierung auf den Vorsatz für den Wandel blind ist, der sich im Bereich minimaler Risiken vom Erfolgsdelikt zum Ungehorsamsdelikt vollzieht (B.I.1. a. E.).

\section{Ergebnis zu „Gestaltungsmacht“}

Im Ergebnis wird der Ansatz (und nur dieser) der beiden genannten Lehren („unabgeschirmte Gefahr“, „Vorsatzgefahr“) hier geteilt: Es geht um die Bestimmung des (vorgestellten) objektiven Tatbestands. Aber zum Zentrum führt weder eine phänotypische Durchführung des Ansatzes (Abschirmung der Gefahr) noch eine Berücksichtigung der Strategien der Erfolgsherbeiführung (Vorsatzgefahr), sondern nur eine Analyse der gesellschaftlichen Praxis, die bereichsweise notwendig zur Gewöhnung an kleinere Überschreitungen der Risikoerlaubnis zwingt und damit solche Überschreitungen aus dem Bereich erfolgsrelevanter und damit entscheidungsrelevanter, „anschaulicher" Gefahrsetzungen ausklammert. Vorsatz ist der Wille mit dem Inhalt, eine entscheidungsrelevante Gefahr zu gestalten. Führt eine ausgeklammerte Gefahrsetzung zu einem Erfolg, so handelt es sich um ein Ungehorsamsdelikt, ${ }^{48}$ nicht um ein Erfolgsdelikt; denn zwar bleibt die generelle Eignung solchen Verhaltens zur Erfolgsherbeiführung praxisrelevant, nicht aber diejenige im Einzelfall. - Die bei gegebener Kenntnis Vorsatz begründende Gefahr ist zwar ein Merkmal des objektiven Tatbestands, steht aber nicht gegen die Fahrlässigkeitsgefahr, sondern gegen die praktisch nicht mehr erfolgsbezogene, abstrakt bleibende Gefahr bloßer Verhaltensregelungen. Es gibt keine reine Fahrlässigkeitsgefahr.

\section{Der Gestaltungsgegenstand des Wollens}

\section{Der Begriff des Delikts}

Der Inhalt des Wollens ist beim Vorsatzdelikt eine Tatbestandsverwirklichung. Üblicherweise wird formuliert, „für einen vorsatzausschließenden Irrtum gemäß $\$ 16$ Abs. 1 StGB“ genüge es, „dass der Täter auch nur einen der Umstände, die den gesetzlichen Tatbestand verwirklichen, nicht kennt, also in Bezug auf nur ein Tatbestandsmerkmal irrt. " ${ }^{49}$ Bei dieser Formulierung werden - übrigens schadlos und in Übereinstimmung mit dem Sprachgebrauch des Gesetzes ( $\$ 16$ Abs. 1 Satz 1 StGB) - die Worte der Tatbestandsformulierung und der jeweilige Begriff des deliktischen

47 Anders Puppe, Vorsatz (Fn. 15), S. 45; dagegen (wie hier) Roxin, Strafrecht AT (Fn. 7), $\mathbb{S} 12$ Rn. $47 \mathrm{ff} . / 51$.

48 Oben B.I.1. (Text zu Fn. 27).

49 LK-Vogel (Fn. 7), $\mathbb{S} 16$ Rn. 17. 
Verhaltens verwechselt. Ein krasses Beispiel: Wenn ein Bauer in der Meinung, in seiner Scheune rumorten nachts Füchse, das Tor abschließt, um die Tiere demnächst im Licht des Morgens erschlagen zu können, während das Geräusch von einem ungebetenen menschlichen Schlafgast stammt, so wäre es falsch zu sagen, er kenne zwar das Merkmal „einsperren“, nicht aber dasjenige „Mensch“ des Tatbestands der Freiheitsberaubung. Im umgekehrten Fall, wenn der Bauer einem Wanderer einen Platz in der Scheune anweist und in der irrigen Meinung, das Haupttor lasse sich problemlos öffnen, die Nebentür verschließt, wäre die Aussage, er kenne zwar das Merkmal „Mensch“, nicht aber dasjenige „einsperren“, nicht minder falsch. „Einsperren“ und „Mensch“ sind nicht per se Tatbestandsmerkmale, sondern aufeinander bezogene Worte zur Bildung eines Begriffs der Freiheitsberaubung ( $\int 239$ StGB). Ebenso wenig lassen sich „Mensch“ und „töten“ trennen ( $\$ 212$ StGB), „fremd“, „Sache“ und „zueignen“ (\$ 246 StGB) etc. Es gibt keine bruchstückhafte Tatbestandskenntnis, was selbst für zusammengesetzte Tatbestände gilt: Kenntnis von einer Nötigung ist nicht partielle Kenntnis von einem Raub ( $\mathbb{S}$ 240, 249 StGB). Die Worte der Tatbestandsformulierung gewinnen nur in ihrer gegenseitigen Bezogenheit Sinn, und gerade diese Bezogenheit muss Gegenstand der Kenntnis sein (was, soweit ersichtlich, auch niemand bezweifelt); ohne diese Bezogenheit geht es um Worte in einem anderen, nicht tatbestandlichen Kontext. Noch krasser: Wer weiß, dass eine Sache fremd ist, hat nicht schon allein deshalb „ein Stück“ der Kenntnis des Diebstahls- oder Sachbeschädigungstatbestands.

Also ist der Begriff des Delikts, das verwirklicht wird, zu kennen: Tötung eines Menschen, Zerstörung einer fremden Sache, Anzünden eines fremden Gebäudes etc. Diese Kenntnis wird prima facie vom Gesetz und der ihm - etwas voreilig - gläubig folgenden Lehre aufgespalten: Die Einsicht in das Unrecht soll etwas anderes sein als die Kenntnis der Tatbestandsverwirklichung, wobei nicht erst an - hier ausgeklammerte - Rechtfertigungslagen zu denken ist, sondern bereits an den Grundfall: einerseits die Kenntnis vom Auslöschen eines menschlichen Lebens, andererseits die Einsicht in das Unrecht dieses Geschehens. Das Gesetz scheint die Trennung für sinnvoll zu halten; denn es knüpft an die Tatbestandsunkenntnis andere Konsequenzen als an ein Fehlen der Unrechtseinsicht ( $\mathbb{S} 16$ Abs. 1 StGB, $\mathbb{S} 17$ StGB). Freilich ergibt ein genauerer Blick, dass die Trennung (1) teils begrifflich unmöglich und auch (2) insgesamt nicht angezeigt ist; (3) jedenfalls ist sie ohne strafrechtlichen Sinn, womit sich zugleich die unterschiedlichen Konsequenzen als korrekturbedürftig erweisen (unten B.II.3.). 


\section{Typen von Tatbestandsmerkmalen}

\section{a. Sogenannte normative Tatbestandsmerkmale ${ }^{50}$}

Die Trennung von Tatbestandskenntnis und Unrechtseinsicht ist bei der Hauptgruppe der sogenannten normativen Tatbestandsmerkmale ${ }^{51}$ unmöglich. ${ }^{52}$ Die normativen Tatbestandsmerkmale zeichnen sich dadurch aus, dass sie als Elemente eines Deliktstatbestands - anders als die sogenannten deskriptiven Merkmale - nicht gegebene Naturtatsachen (genauer, was die Gesellschaft als solche versteht: „Au-

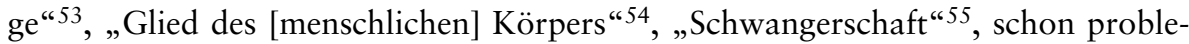
matisch: „Wälder" $\left.{ }^{56}\right)$ bezeichnen, sondern Ergebnisse der normativen Strukturierung der Gesellschaft und in diesem Sinn soziale Tatsachen. ${ }^{57}$ Die Struktur kann was hier weniger interessiert - durch außerrechtliche Normen gebildet werden, auf die sich das Recht bezieht und die dadurch in das Recht inkorporiert werden, ohne dass sich an ihrer außerrechtlichen Genese etwas ändert („Handelsbrauch“, möglicherweise, je nach dem Zusammenhang, „Lebensgemeinschaft“, „Familie“, „Straße“), oder aber - und darauf kommt es hier an - durch rechtliche Normen, und die sich dann ergebenden Merkmale bilden die Hauptgruppe: Eine rechtliche Tatsache $^{58}$ (Eigentum, Zuständigkeit zur Eidesabnahme, Amtsträger) wird von ihrer strafrechtlich-tatbestandlichen Seite her dargestellt. Hierher gehören auch die Merkmale, die zwar umrisshaft auf außerrechtliche Normen, insbesondere solche der Sitte, bezogen sind, bei denen sich aber das Recht die Konkretisierung und Weiterentwicklung vorbehält, die also vom Recht als Rechtsnormen inkorporiert werden: bereichsweise die Institutionen der Lehre vom unerlaubten Verhalten („objektive Zurechnung “), ferner, ebenfalls bereichsweise, Garantenpflichten.

Das idealtypische normative Tatbestandsmerkmal ist „fremd“ („fremde Sache“) bei den Eigentumsdelikten. „Fremd“ ist eine Sache, wenn ein anderer als der Täter Ei-

50 Blankettmerkmale sind entweder normative Tatbestandsmerkmale oder verweisen auf Ergänzungen des Tatbestands eines Ungehorsamsdelikts (Jakobs, Strafrecht AT (Fn. 10), Abschn. 8 Rn. 47). Beispielhaft, in diesem Fall sind die Fahrer von rechts kommender Fahrzeuge vorzulassen, in jenem vorfahrtsberechtige Fahrer. Die Unterscheidung wird hier nicht weiter verfolgt.

51 Eingehend T. Walter, Der Kern des Strafrechts. Die allgemeine Lehre vom Verbrechen und die Lehre vom Irrtum, Tübingen 2006, S. 389 ff./393 ff., irrtumstheoretisch S. 217 ff.

52 Immerhin an der Trennbarkeit der Kenntnisgegenstände bei normativen Merkmalen zweifelnd LKVogel (Fn. 7), $\$ 16$ Rn. 31 ff.

$53 \int 226 \mathrm{StGB}$.

$54 \int 226 \mathrm{StGB}$.

$55 \mathbb{\$} 218 \mathrm{ff}$. StGB.

56 \306 StGB.

57 Es ist nicht sachgerecht, den „Wertungsirrtum“ bei diesen Tatsachen einem vom Tatbestandsirrtum abgetrennten Unrechtsirrtum mit der Begründung zuzuweisen, die Flexibilität von $\mathbb{1} 17$ StGB garantiere angemessene Ergebnisse; so aber Rinck, Der zweistufige Deliktsaufbau, Berlin 2000, S. 346 f. 472. Der dann verbleibende Kenntnisrest lässt sich nicht als Kenntnis eines Unrechtssubstrats darstellen.

58 Institutionelle Tatsachen als Konstitutionselemente der gesellschaftlichen Wirklichkeit, J. Searle, Die Konstruktion der gesellschaftlichen Wirklichkeit, Reinbek 1997, S. 41 ff., 69 ff.; ders., Geist, Sprache und Gesellschaft, Frankfurt am Main 2001, S. 134 ff. 
gentümer ist, und was Eigentum ausmacht, regelt $\mathbb{\$} 903$ BGB: Damit darf der Berechtigte nach Belieben verfahren und sonst niemand. Wenn der Täter die soziale Tatsache „fremdes Eigentum“ kennt, so weiß er auch, dass die Sache von ihm weder unterschlagen noch gestohlen noch beschädigt oder zerstört werden darf und weiteres mehr nicht, für das es keinen generellen Straftatbestand geben mag, wie etwa für das bloße Entziehen der Sache (furtum usus). Mit anderen Worten, mit der Kenntnis, dass und worauf er - außerhalb einer rechtfertigenden Situation - zugreift, kennt der Täter das Unrecht des Zugriffs, und das lässt sich umkehren: Kennt der Täter das Unrecht nicht, so ist ihm auch die Tatbestandsverwirklichung nicht bekannt oder er nimmt eine rechtfertigende Situation an. Beispielhaft, wer weiß, dass er eine fremde Sache außerhalb einer Rechtfertigungslage zerstört, kennt auch das Unrecht seines Tuns, ${ }^{59}$ und wer dieses nicht erkennt, weiß auch nichts von der Fremdheit oder nichts von der zerstörerischen Kraft seines Vorgehens, oder es liegt seines Erachtens eine Rechtfertigungslage vor.

Nun nennt das Recht zwar bei den Eigentumsdelikten die rechtliche Qualifikation des Gestaltungsgegenstands, eben die Fremdheit der Sache, aber etwa bei den Tötungsdelikten nennt es nichts dergleichen, sondern formuliert den Tatbestand als „Tötung eines Menschen“, und wer einen anderen Menschen völlig als sein Eigentum begriffe, als einen rechtlosen Sklaven, könnte ihn töten, also den genannten Tatbestand verwirklichen, ohne damit auch das Unrecht seines Verhaltens einzusehen. Aber eine solche Lesart der Tötungsdelikte wäre, zumal mit Blick auf - etwa - die Eigentumsdelikte, befremdlich: Warum sollte bei diesen der Eingriff in das Recht eines anderen zum Tatbestand gehören, bei jenen hingegen nicht, vielmehr allein auf das factum brutum der Auslöschung eines Menschenlebens abzustellen sein? Dem ist auch nicht so; vielmehr nennt das Gesetz bei den Tötungsdelikten den Rechtseingriff nicht, weil er, anders als bei einem Eingriff in eigentumsfähige Sachen, selbstverständlich ist oder doch sein sollte. Eine Sache mag einer anderen Person oder dem Täter gehören oder herrenlos sein, aber das menschliche Leben „gehört“ stets demjenigen, der lebt, keinem anderen und auch nicht niemandem. Das Recht auf Leben ist nach heutigem Verständnis mit der Benennung als „Mensch“ mit benannt, und weil demgemäß bei den Tötungsdelikten der Mensch als Rechtsinhaber gemeint ist, gilt der tötende Eingriff dem Menschen als Person. ${ }^{60}$ Im zuvor genannten Beispiel hätte der Täter also nicht allein das Unrecht seiner Tat nicht eingesehen, vielmehr

59 A. A. insbesondere I. Puppe, Vorsatz und Rechtsirrtum, in: H. Putzke u. a. (Hrsg.), Strafrecht zwischen System und Telos. Festschrift für Rolf Dietrich Herzberg, Tübingen 2008, S. 275 ff.; dies., Tatirrtum, Rechtsirrtum, Subsumtionsirrtum, GA 1990, 145 (154 ff./157 Fn. 24). Puppe will zwischen dem „außerstrafrechtlichen Sinn“ eines „Rechts oder Rechtsverhältnisses“ und „dem strafrechtlichen Gebot, es nicht zu verletzen, “ unterscheiden, weil die Tatbestände ansonsten „tautologisch“ "seien (Vorsatz, S. 297). Letzteres sollte nicht schrecken: Das siebente Gebot ist in der Tat nur ein Auszug aus der Festsetzung „Es sei Eigentum“!

60 Zur Person als Inhaberin von Rechten und Pflichten siehe I. 1. $\$ 1$ ALR; G. Jakobs, Norm, Person, Gesellschaft, 3. Auflage, Berlin 2008, S. 28 ff./31 ff. 
bereits missverstanden, worauf er zugreift, nämlich auf eine Person, und demgemäß würde ihm die Kenntnis fehlen, den - richtig gelesenen! - Tatbestand zu verwirklichen.

Nicht anders verhält es sich bei der Freiheitsberaubung ( $\$ 239$ StGB) oder bei der Nötigung ( $\$ 240$ StGB): Formuliert wird „Mensch“, aber zu lesen ist „Person“ “61 als Inhaberin des Rechts auf Freiheit, ${ }^{62}$ worüber das Gesetz keine eigenen Worte verliert, weil die Rechtszuständigkeit bei höchstpersönlichen Delikten - anders als bei Eigentumsdelikten - immer nur bei dieser einen Person liegen kann. Beispielhaft, wer meint, einem Sklaven seine „Freiheit“ zu nehmen, versteht bereits den Tatbestand der Freiheitsdelikte nicht und verkennt nicht nur das Unrecht einer ihrerseits gekannten Tatbestandsverwirklichung. Entsprechendes könnte für sämtliche Verletzungs- oder konkreten Gefährdungsdelikte dargetan werden: Ein Begreifen des Eingriffs ist ohne Begreifen des Unrechts ausgeschlossen. ${ }^{63}$ Ein solches Begreifen scheidet freilich bei den Ungehorsamsdelikten, insbesondere in der Gestalt abstrakter Gefährdungsdelikte, aus, wovon noch zu handeln sein wird (unten B.II.3.).

\section{b. Merkmale der objektiven Zurechenbarkeit}

Die Kenntnis eines Eingriffs in die Rechte einer Person als Kenntnis des Unrechts findet ihre Entsprechung in der Kenntnis des Täters (oder des Beteiligten) von der objektiven Zurechenbarkeit seines Verhaltens. Beispielhaft, wenn der Täter weiß, dass beim Verleihen einer scharfen Axt an seinen Nachbarn, der die Schärfe kennt, alles Weitere auf dessen Gefahr geschieht, er, der Täter, also nicht für eine (Selbst-) Verletzung des Nachbarn zuständig wird (und das ist der Gehalt des normativen Ausschlussmerkmals „Handeln auf eigene Gefahr“, ein negatives Tatbestandsmerkmal), so setzt dieses Wissen die Ordnung der sozialen Welt nach Organisationskreisen voraus und damit das Wissen um das Unrecht eines Eingriffs in die fremde Organisation. Gewiss muss der Täter nicht theoretisieren, er selbst könne zuständig

61 Nur das Verständnis des Menschen als Person ermöglicht eine konsistente Auslegung der Erpressung $(\$ 253 \mathrm{StGB})$ : Der Schaden muss eine Person treffen, nicht einen Menschen (nur Personen haben Rechte, die „beschädigt“ werden können), und diese Person kann, wie der Eigentümer bei den Eigentumsdelikten, auch eine juristische Person sein. R. Wallau, Der „Mensch“ in $\mathbb{S}$ 240, 241, 254 StGB und die Verletzung der Rechte juristischer Personen, JR 2000, S. 312 ff.

62 Damit steht zugleich fest, dass Nötigungsmittel (auch bei der Erpressung) nur sein kann, was den Genötigten in seinem Recht auf Freiheit beschränkt. Etwa die „Drohung“ mit einem erlaubten Verhalten lässt sich im Rahmen eines Freiheitsdelikts (anders etwa bei einem Wucherdelikt) nicht unterbringen. G. Jakobs, Nötigung durch Drohung als Freiheitsdelikt, in: J. Baumann u. a. (Hrsg.), Einheit und Vielfalt des Strafrechts. Festschrift für Karl Peters, Tübingen 1974, S. 69 (72 ff./85 ff.); dazu SKWolter/Horn (Fn. 7), \$2 40 Rn. 7 mit Nachweisen.

63 Zwei Andeutungen: (1) Beim Betrug (\$2 263 StGB) geht es nicht um irgendeine Täuschung mit schädlichen Folgen für das Vermögen, sondern nur um die Verletzung des Rechts, in Vermögensangelegenheiten nicht belogen zu werden, und darauf muss sich der Vorsatz des Täters beziehen. Außerhalb dieses Rechts, etwa bei der Täuschung über die eigene Gewinnerwartung, ist im Grundfall (ohne Bestand einer besonderen Garantenstellung) Betrug ausgeschlossen. Grundlegend Pawlik, Das unerlaubte Verhalten beim Betrug, 1999, S. $65 \mathrm{ff.} / 139 \mathrm{ff}$. - (2) Bei der falschen (eidlichen) Aussage ( $\mathbb{S} 153 \mathrm{ff}$. StGB) „steckt“ in der „zuständigen Stelle“ deren Recht auf eine wahre (wahrhaftige) Aussage. 
werden (bei der Überschreitung des erlaubten Risikos) oder eine dritte Person (beim Vertrauensgrundsatz oder beim Regressverbot) oder das Opfer selbst (eben beim Handeln auf eigene Gefahr und bei casus), aber er muss doch wissen, dass eine Fremdverwaltung von Organisationskreisen, die anderen Personen zustehen, von Rechts wegen nicht sein soll, er sich also bei einem Eingriff etwas ihm selbst nicht Zustehendes anmaßt. Dieses Wissen ist Unrechtseinsicht!

\section{c. Garantenstellungen}

Entsprechendes gilt für die Kenntnis von Garantenstellungen: ${ }^{64}$ Eine Kenntnis der Pflichtvoraussetzungen von der Kenntnis der Pflicht selbst zu trennen, ${ }^{65}$ ist nicht möglich, solange man die Voraussetzungen als Ereignisse in einer normativ strukturierten Gesellschaft und nicht als ein Sammelsurium „nackter“ Tatsachen versteht. Beispielhaft, wer weiß, dass er Vater, Mutter oder Polizist etc. ist, kennt auch seine besonderen (nicht jedermann obliegenden) ${ }^{66}$ Pflichten gegenüber dem Schutzbefohlenen, und wer weiß, dass er ein Sonderrisiko in einen fremden Organisationskreis „hineingetragen“ hat (Ingerenz), kennt auch seine Pflicht zur Vermeidung eines Schadens; denn ansonsten ginge er nicht von der Besonderheit des Risikos aus, vielmehr von einem ubiquitären, vom Opfer selbst oder von dritten Personen zu tragenden Risiko. ${ }^{67}$

\section{d. Appellfunktion des Willensinhalts. Zugleich Ergebnis zu „Gestaltungsgegenstand“}

Wenn beim Kernbestand der Tatbestände aus mehreren, sich teils überschneidenden Gründen die Tatbestandskenntnis von der Unrechtseinsicht nicht geschieden werden kann, es also um die Beschreibung von Rechtsverletzungen geht, die der Vorsatztäter als solche begriffen haben muss, besteht kein Grund, es im Randbereich, nämlich bei Tatbestandsformulierungen, die sich in der Beschreibung eines bestimmten Verhaltens erschöpfen, für den Vorsatz bei der Kenntnis des Beschriebenen zu belassen: Die Kenntnis eines Sammelsuriums an Fakten muss für den Täter bedeutungslos bleiben, solange es eben um ein Sammelsurium geht und nicht um eine Ordnung, wenn also kein Begriff eines Delikts dazukommt. Der Täter muss nicht erst einmal „etwas“ an Fakten wissen, um beurteilen zu können, ob es sich dabei um Unrecht handelt; denn vor dem Unrechtsurteil weiß er überhaupt nicht, auf welche Fakten es ankommt.

64 Gleichfalls für die Kenntnis gesamttatbewertender Merkmale; G. Jakobs, Dolus malus, in: K. Rogall u. a. (Hrsg.), Festschrift für Hans-Joachim Rudolphi, Berlin et al. 2004, S. 107 (118 ff.).

65 So BGHSt 16, 155 (158), wo diese Trennung nur gelungen sein dürfte, weil es - entgegen dem BGH - an einer Garantenstellung fehlte: Aus den vom Täter gekannten Voraussetzungen ergibt sich keine Pflicht. - Wie der BGH entscheidet auch die überwiegende Lehre; siehe nur Schönke/Schröder-Cramer/ Sternberg-Lieben (Fn. 7), $\mathbb{1 5}$ Rn. 93 ff., 96 mit eingehenden Nachweisen.

66 Zu diesen Pflichten: L. Sánchez-Vera, Pflichtdelikt und Beteiligung, zugleich ein Beitrag zur Einheitlichkeit der Zurechnung bei Tun und Unterlassen, Berlin 1999, S. 76/102 ff. und passim.

67 Das gilt auch für Begehungsdelikte; zur Notwendigkeit einer (wegen der Verkehrssicherungspflicht für den eigenen Körper praktisch selten fehlenden) Garantenstellung bei diesen Delikten Jakobs, Strafrecht AT (Fn. 10), Abschn. 7 Rn. 56 ff. 
Welche - unterstellt - fünf von fünfundzwanzig in einer Situation gekannten Fakten soll der Täter zu einer rechtlichen, appellativen Sinneinheit zusammenstellen, wenn er die Auswahlregeln, nämlich die Regeln der Unrechtsbestimmung und -vermeidung nicht kennt? Wenn bei der Faktenkenntnis nicht ein „Platzhalter“ (in der Regel ein normatives Tatbestandsmerkmal) auf das Unrecht verweist, ist die oft herangezogene „Appellfunktion“ des Tatbestandsvorsatzes eine reine Schimäre. Also muss in per se neutrale Verhaltensbeschreibungen (bei Ungehorsamsdelikten und abstrakten Gefährdungsdelikten) dieser „Platzhalter“ in die Tatbestandsbeschreibung hineingelesen werden. ${ }^{68}$

Dass die Abspaltung einer Tatbestandskenntnis von der Unrechtseinsicht unmöglich oder doch untunlich ist, verbindet sich systematisch mit der mittlerweile nicht mehr nur vereinzelt vertretenen Lehre, die im Strafrecht die Rechtswidrigkeit neben der Schuld allenfalls als didaktische (bei der Begriffsentwicklung hilfreiche) Stufe berücksichtigt, nicht aber als eigenständige strafrechtliche Beurteilung: Strafrecht reagiert auf Rechtsuntreue, also auf Schuld, ${ }^{69}$ und bei der Rechtswidrigkeit geht es deshalb um eine Vorfrage, nicht um mehr. ${ }^{70}$ Die Beantwortung dieser strafrechtlichen Vorfrage mag für andere Rechtsfolgen als die Strafe eine Antwort auf die dort maßgebliche Hauptfrage geben. Beispielhaft, gegen einen seelisch kranken Menschen, dem aber zugestanden wird, zumindest einige seiner Rechte (Leib!) „frei“ zu

68 K. Tiedemann, Tatbestandsfunktionen im Nebenstrafrecht, Tübingen 1969, S. 372 ff.; Jakobs, Dolus malus (Fn. 64), S. 119 ff.; M. Pawlik, Strafrechtswissenschaftstheorie, in: ders. u. a. (Hrsg.), Festschrift für Günther Jakobs, Köln et al. 2007, S. 469 (488 ff.) - Zum Meinungsstand mit der Option für eine „weichere“ Schuldtheorie C. Roxin, Über Tatbestands- und Verbotsirrtum, in: U. Sieber u. a. (Hrsg.), Strafrecht und Wirtschaftsstrafrecht, Festschrift für Klaus Tiedemann, Köln et al. 2008, S. 375 (388 ff.).

69 M. Pawlik, „Der wichtigste dogmatische Fortschritt der letzten Menschenalter?“. Anmerkungen zur Unterscheidung von Unrecht und Schuld im Strafrecht, in: G. Dannecker u. a. (Hrsg.), Festschrift für Harro Otto, Köln et al. 2007, S. 133 (148f.); G. Jakobs, Der strafrechtliche Handlungsbegriff, Berlin 1992, S. 41 ff.; Walter, Kern (Fn. 51), S. 81 ff./196 ff. und passim; H. Lesch, Der Verbrechensbegriff. Grundlinien einer funktionalen Revision, Köln et al. 1999, S. 184 ff./190 ff./203 ff.; A. Sinn, Straffreistellung aufgrund von Drittverhalten. Zurechnung und Freistellung durch Macht, Tübingen 2007, S. $271 \mathrm{ff} . / 287$ u. a. m. Dagegen insbesondere L. Greco, Wider die jüngere Relativierung der Unterscheidung von Unrecht und Schuld, GA 2009, S. 636 ff. mit Nachweisen; C. Roxin, Gedanken zur strafrechtlichen Systembildung, in: Silva Dias u. a. (Hrsg.), Liber Amicorum de José de Sousa e Brito, Coimbra (Almedina) 2009, S. 777 (785 f.).

70 Diese Vorfrage erledigt sich, was die subjektive Seite der Angelegenheit betrifft, nicht etwa nach anderen Prinzipien als die strafrechtliche Hauptfrage. Entgegen Welzel lässt sich nicht begründen, dass im Unrechtsbereich ein „Kenntnisprinzip“ gelten soll, im Schuldbereich hingegen ein „Verantwortungsprinzip“ (H. Welzel, Das Deutsche Strafrecht, 5. Auflage, Berlin 1956, S. 134; 11. Auflage, Berlin 1962, S. 162; dagegen G. Jakobs, Handlungssteuerung und Antriebssteuerung - Zu Hans Welzels Verbrechensbegriff, in: Amelung u. a. (Hrsg.), Strafrecht. Biorecht. Rechtsphilosophie, Festschrift für Hans-Ludwig Schreiber, Heidelberg 2003, S. 949 (952 ff./955 ff.)). Ein „Prinzip“, das es bei einem potenziell Verantwortlichen honoriert, wenn er vor dem Kontext seiner Pläne die Augen verschließt („Kenntnisprinzip“), gelang selbst zur Blütezeit des Psychologismus (Was sieht der Täter?), als dieser noch mit einer Straftheorie verbunden war (also etwa bei P. J. A. von Feuerbach, Revision der Grundsätze und Grundbegriffe des positiven peinlichen Rechts, I. Theil, 1799, S. 44 ff./186, II. Theil, 1800, S. 43 ff; dazu Lesch, Verbrechensbegriff (Fn. 69), S. 44 ff./57 ff.), nicht recht (siehe Feuerbachs Schwierigkeiten in Fällen von culpa, Lehrbuch des gemeinen in Deutschland gültigen peinlichen Rechts, 11. Auflage, 1832, $\mathbb{S}$ S55-59 und zur culpa dolo determinata $\$ 60)$. 
verwalten, kann nach den Regeln des defensiven Notstands vorgegangen werden, ${ }^{71}$ wenn diese nur instrumentelle ${ }^{72}$ Verwaltung für andere gefährlich wird. Dass sich zu einem solchen Fall in jedem Strafrechtslehrbuch etwas findet, heißt nicht, er sei per se auch unmittelbar strafrechtlich relevant.

\section{Nur präsentes Wissen?}

\section{Wertungsfehler versus Wissensfehler}

Der Vorsatztäter wie der Fahrlässigkeitstäter handeln rechtsuntreu, und zwar der Vorsatztäter, indem er seine Zweckverfolgung höher stellt als die Vermeidung eines Delikts, der Fahrlässigkeitstäter, indem er nicht hinreichend um die Schadlosigkeit seines Verhaltens besorgt ist (oder wenn ihm ein weiteres „Besorgen“ die Sache nicht wert ist, seine Handlung „vorsichtshalber“ nicht vollzieht). Beide Täter wollen also, was sie nicht wollen dürfen: Ihre Rechtsuntreue beruht auf einem Wollensfehler. Während jedoch beim Vorsatztäter der Wollensfehler durch eine offen fehlerhafte, eben unrechtliche, Wertung der zutreffend erkannten („natürlichen“ und institutionellen) Tatsachen zustande kommt, verbirgt beim Fahrlässigkeitstäter die verkürzte Kenntnis der Tatsachenbasis das Fehlerhafte des Willensinhalts, und dieser wird vom Täter nicht als inkorrekt erkannt; - schlagworthaft: Wertungsfehler versus Wissensfehler. ${ }^{73}$

Der Fehler des Fahrlässigkeitstäters ließe sich als der schwerere gewichten: Der Täter weiß vermeidbar (!) nicht, was er anrichtet, und das kann „alles Mögliche“ sein, ${ }^{74}$ jedenfalls wird der Umfang der Verhaltensfolgen nicht auf das zur - wenn auch einigermaßen rücksichtslosen - Zweckverfolgung Erforderliche begrenzt. Beispielhaft, ${ }^{75}$ eine halbe Sekunde Unachtsamkeit im Flug- oder selbst im Straßenverkehr kann mehr Menschenleben kosten, als mancher sogenannte Massenmörder in der Zeit seines „Wirkens“ zusammenbringt. Wenn das geltende Recht die Tatfahrlässigkeit trotz der potenziellen Uferlosigkeit der Folgen milder beurteilt als den Tatvorsatz (was üblicherweise geradezu selbstverständlich als Plus-Minus-Verhältnis

71 G. Jakobs, Rechtszwang und Personalität, Paderborn 2008, S. 20 ff. mit Nachweisen.

72 Finalität, die von der Schuld abstrahiert, belässt nur eine „instrumentelle“ Steuerung; dazu Jakobs, Handlungssteuerung (Fn. 70), S. $950 \mathrm{ff}$.

73 Diese Bezeichnung ziehe ich der früher von mir vorgeschlagenen vor: Wollensfehler versus Wissensfehler (G. Jakobs, Wollensfehler und Wissensfehler, ZStW 101 (1989), S. 516 ff.); denn auch der Fahrlässigkeitstäter weist einen Wollensfehler auf. Nur die - zuvor unglücklich gewählte - Terminologie ändert sich.

74 Dass die Gefährlichkeit des Fahrlässigen „für die Rechtsordnung nur eine zufällige und infolgedessen eine verminderte“ sei (B. Schünemann, Vorsatzbegriff (Fn. 35), S. 373), ist nicht richtig: Bei gegebenem Vorsatz nebst Unrechtseinsicht werden Folgen einer Vorsatztat immerhin durch die Bewertung des Täters gefiltert; nur selten handeln Täter „um jeden Preis“. Beim Fahrlässigen wird nichts durch einen Filter aufgehalten.

75 Nach LK-Vogel (Fn.7), $\mathbb{1} 15$ Rn. 10. 
hingenommen wird), ${ }^{76}$ so geht es dabei weder um Tatherrschaftsdifferenzen ${ }^{77}$ noch, ins Subjektive gewendet, um die leichtere Vermeidbarkeit der Vorsatztat und schon gar nicht um die „Kosten“ der Vermeidung: ${ }^{78}$ Dass etwa Aufmerksamkeit auf die körperliche Integrität der anderen schwerer (oder nur mit größeren „Kosten“) zu leisten sei, als der Verzicht darauf, einen frechen Nebenbuhler zu verprügeln, setzt voraus, was zu begründen wäre: die leichtere („kostengünstigere“) Vermeidbarkeit eines Willensfehlers. Mögen solche Überlegungen auch bei der Gesetzesfassung Pate gestanden haben, so bilden sie doch nicht den objektiven Grund der Regelung. Dieser ist - strafrechtsfunktional - an anderer Stelle zu suchen.

Ein Wissensfehler wirkt in eine nicht vorab bestimmte Richtung, also eventuell auch gegen den Täter selbst, der deshalb durch seine Tat die Gefahr einer poena naturalis heraufbeschwört. Beispielhaft, bei einer Kollision von Kraftfahrzeugen wegen der Unaufmerksamkeit eines der Fahrer wird auch dieser selbst nur selten ungeschoren davonkommen. Da der Täter zudem in der Regel kein sonderliches Interesse gerade an der Unaufmerksamkeit beim Vollzug seines Verhaltens hat, befinde er sich nun in Tat- oder in Rechtsfahrlässigkeit, wird der „Ärger“, der sich zumindest nach einer folgenreichen Fahrlässigkeitstat einzustellen pflegt (peinliche Untersuchung, Bestrafung, Schadensersatzpflicht), durch keinen Vorteil kompensiert. Kurzum, jede Person wird sich im Eigeninteresse bemühen, Fahrlässigkeitstaten zu vermeiden; mehr noch, diese Taten offenbaren einen Mangel an Kompetenz zur Selbstverwaltung, so dass die Bestrafung die ohnehin auf der Hand liegende mangelnde Anschlussfähigkeit der Tat - niemand hält Desorientierung für einen erstrebenswerten Zustand - nur nachzeichnen muss. ${ }^{79}$ Hingegen erweist sich ein Wertungsfehler, also der Fehler eines Vorsatztäters, nicht eo ipso als für das Individuum falsch: Manches Imperium wurde durch ein Verbrechen begründet (aber wohl keines durch unsorgfältiges Verhalten). - Das Recht kann die Vorzugswürdigkeit seiner Befolgung nicht so beweisen, wie die Vorzugswürdigkeit von Sorgfalt beweisbar ist. Deshalb bleibt dem Recht nur ein Weg, diese Vorzugswürdigkeit in der Kommunikation zu etablieren: Es muss dem Täter aufgeben, selbst Gründe für die Rechtsbefolgung zu beschaffen. Gelingt das dem Täter nicht, so ist das sein Fehler, strafrechtlich: seine Schuld. In anderen nor-

76 Zum Diskussionsstand über den Grund der Wertungsdifferenz C.-F. Stuckenberg, Vorstudien zu Vorsatz und Irrtum im Völkerstrafrecht. Versuch einer Elementarlehre für eine internationale Vorsatzdogmatik, Berlin 2007, S. 406 ff.; LK-Vogel (Fn. 7), \$15 Rn. 8 ff.; Jakobs, Strafrecht AT (Fn. 10), Abschn. 8 Rn. 5 ff.

77 Schünemann, Vorsatzbegriff (Fn. 35), S. 371: „Mißbrauch qualifizierter Vermeidemacht“ (aber diese Macht ist reine Faktizität und wird nach ihrer objektiven Seite auch von demjenigen missbraucht, der nicht merkt, dass er sie gebraucht); von dem Gedanken der Vermeidemacht abrückend aber S. 372: Entscheidend sei die „Gesinnung“ als „, die durch das bewußte Handeln des Täters manifestierte Maxime seines Handelns. “ Das nähert sich dem hier herausgestellten Wertungsfehler an, der freilich mit einer „Gesinnung“ nicht gleichgestellt werden sollte.

78 M. Adams/S. Shavell, Zur Strafbarkeit des Versuchs, GA 1990, S. 337 (356f.) - eine einigermaßen offen liegende petitio principii (siehe den Text).

79 Jakobs, Wollensfehler (Fn. 73), S. $527 \mathrm{ff}$. 
mativen Systemen verhält es sich ebenso; ein Hinweis auf mangelnde „Lust“ zur Normbefolgung kann weder im Recht noch etwa in der Moral entlasten, ein solcher auf Desorientierung aber sehr wohl. Erneut schlagworthaft: Wertungsfehler entlasten nicht, wohl aber Wissensfehler.

\section{Dolus indirectus ${ }^{80}$}

Die übliche Lehre kommt mit der oben erwähnten Annahme eines Plus-Minus-Verhältnisses (II. C. 1.) bei gegebenem Wissen zum gleichen Ergebnis; da sie aber Wertungs- und Wissensfehler nur als vermeintlich psychische Quantitäten, nicht qualitativ gegenüberstellt, führt sie zu Fehlern, wenn ein Nicht-Wissen (Wissens-,,Fehler“) auf einem Wertungsfehler beruht: ${ }^{81}$ In einer solchen Situation sieht der Täter bereichsweise nicht, was er anrichtet und wie das Recht die Lage beurteilt (Nichtwissen), weil das für ihn gleichgültig, nicht entscheidungsrelevant ist (Wertungsfehler). ${ }^{82}$ Beispielhaft, ein Laborleiter, der auf Grund seiner Erfahrung längst nicht mehr mit staatlichen Kontrollen rechnet, lässt unbekümmert seine Mitarbeiter diverse Substanzen in ein Gewässer leiten, ohne sich deren Wirkung und die Rechtslage aktuell zu vergegenwärtigen. Oder, ein Täter sticht ein langes Messer tief in den Leib seines Opfers, ohne dass er in seiner Gleichgültigkeit die Lebensgefahr psychisch realisieren würde. Was die fehlende psychische Präsenz des rechtlichen Anspruchs angeht, lässt sich das Problem nach $\mathbb{} 17$ StGB im Ergebnis befriedigend behandeln: ${ }^{83}$ Die Strafe muss nicht gemildert werden (ausgehend von einer Strafe für eine

80 Eingehend G. Jakobs, Gleichgültigkeit als dolus indirectus, ZStW 114 (2002), S. 584 ff.; zur neueren Geschichte Puppe, Vorstellungsinhalt (Fn. 35), S. 23-31; NK-Puppe (Fn. 10), \15 Rn. $14 \mathrm{ff}$; Stuckenberg, Vorstudien (Fn. 76), S. 539 ff./557 ff. und passim; Lesch, Verbrechensbegriff (Fn. 69), S. $61 \mathrm{ff} . / 140 \mathrm{ff}$ (dolus indirectus bei Hegel). - Siehe zudem M. Heuchemer, Der Erlaubnistatbestandsirrtum, Berlin 2005, S. 275 ff.; Y.-A. Hsu, „Doppelindividualisierung“ und Irrtum. Studien zur strafrechtlichen Lehre von der Erfolgszurechnung zum Vorsatz, Tübingen 2007, S. 128 ff./221 ff.; M. Pawlik, Person. Subjekt. Bürger, Berlin 2004, S. 85 ff.

81 C. Burchard, Irren ist menschlich. Vorsatz und Tatbestandsirrtum im Lichte der Emanzipation des angegriffenen Mitmenschen, Tübingen 2008, behandelt Fälle, in denen ein Vorsatztäter über die genauen Tatfolgen falsche Vorstellungen hat, und will demjenigen, der ,sich zu Gewalt gegen seine Mitmenschen versteigt“, das „allgemein Menschen- oder Erfahrungsmögliche als sein Werk“ zuschreiben (S. 384/494f.): Der Vorsatztäter soll damit rechnen müssen, dass er als Mensch nur irrtumsanfällig handeln kann. Das ist für den Bereich des dolus indirectus (der nach hiesigem Verständnis freilich keine aktuelle Kenntnis von einem Eingriff bei anderen voraussetzt, Jakobs, Gleichgültigkeit (Fn. 80), S. 593 ff.) plausibel, aber auch nur dort. Ansonsten mangelt es gegenüber dem eintretenden Geschehen an einem Wertungsfehler. Eingehender zu Burchard: G. Jakobs, Buchbesprechung, HRRS 11/2009, S. $456 \mathrm{ff}$.

82 Rinck unterscheidet beim subjektiven Tatbestand Fälle von (1) Kenntnis, (2) irriger Annahme, die Tatbestandsmerkmale seien nicht gegeben, und (3) einem Nicht-Bedenken der Lage, (Fn. 57), S. 371. Die letztgenannte Gruppe will er dem Vorsatz zuschlagen (S. $381 \mathrm{ff} . / 383 \mathrm{ff} . / 473$ ). Diese Lösung hat einen partiell plausiblen Ansatz: Nicht-Bedenken kann die Folge eines Wertungsfehlers sein, eben Folge von Gleichgültigkeit, muss es aber nicht sein, etwa wenn der Täter wegen eines Schreckens oder wegen plötzlicher Überlastung auf den Kontext seines Verhaltens nicht hinreichend achtet. Rinck beachtet also die Restriktionen der Lehre vom dolus indirectus nicht.

83 Die Regelung wird freilich nicht selten als „zu hart“ beurteilt, siehe Walter, Kern (Fn. 51), S. 410 ff., K. Gaede, Auf dem Weg zum potentiellen Vorsatz? Problematik und Berechtigung der zunehmenden Tendenzen zur normativen Relativierung des Vorsatzerfordernisses, ZStW 121 (2009), S. 239 (241 und öfter, jeweils mit Nachweisen); tendenziell ebenso NK-U. Neumann (Fn. 10), $\$ 17$ Rn. 83 
Tat in aktueller Unrechtseinsicht), wenn auch die Bezeichnung des Nichtwissens als „Irrtum“ in der genannten Vorschrift von Sprachvergessenheit zeugt: Irren kann nur, wer meint, an das Richtige anzuschließen, und das „meint“ der Gleichgültige gerade nicht; abermals beispielhaft, niemand irrt sich über die Uhrzeit, solange ihm - vielleicht bei fesselnder Lektüre - keine Stunde schlägt.

Wenn das Recht die Bedingungen seiner Geltung garantieren soll, kann es Gleichgültigkeit ihm gegenüber nicht mit einem Strafnachlass honorieren; das ist die objektive Aussage von $\mathbb{} 17$ StGB. Die Theorie hat diesen Grundsatz eindeutig zu entfalten, auch wenn seine praktische Bedeutung nicht sonderlich groß sein mag. ${ }^{84} \mathrm{Ge}-$ gen die Gleichstellung der aus Gleichgültigkeit resultierenden Unwissenheit, die hier dolus indirectus genannt wird, mit dem dolus directus und eventualis wird vorgebracht, im Bereich des Tatvorsatzes, also in dem nach allgemeinem Verständnis durch $\mathbb{1} 16$ Abs. 1 Satz 1 StGB geregelten Bereich, bestehe für einen Täter „ohne ... Faktenwissen ... kein Anlass, das Vermeidemotiv zu bilden und eine Alternative des konkreten Handelns zu ergreifen“. ${ }^{85}$ Vielmehr müsse „der Täter (bei Vorsatztaten; G.J.) die Tatbestandsverwirklichung in einem solchen Maße für wahrscheinlich halten, dass es für einen rechtstreuen Normadressaten praktisch notwendig wäre, die Intention zur Vermeidung der Tatbestandsverwirklichung zu bilden. "86 Diese auf präsentes Wissen bezogene Argumentation geht am Problem vorbei: Allemal soll mindestens wegen Fahrlässigkeit zu strafen sein, aber auch der Fahrlässigkeitstäter benötigt einen „Anlass“, 87 für den jedoch hinreicht, dass er auf den Vollzug seiner Handlung verzichtet hätte, wenn er um die Vermeidung von deren Folgen - aus welchen Gründen auch immer! - besorgt gewesen wäre. „Anlass“ im Bereich des Tat-

(und 55); wie hier MK-Joecks (Fn. 7), $\mathbb{S} 17$ Rn. 68. - Für den Erlaubnistatbestandsirrtum stellt Heuchemer konsequent, also unter Verwerfung aller psychologisierenden Argumente (Erlaubnistatbestandsirrtum (Fn. 80), S. $265 \mathrm{ff} . / 275 \mathrm{ff} . / 294 \mathrm{ff} . / 350 \mathrm{ff}$. und passim), auf einen Wertungsfehler ab (S. 286). Nachdem er für den von ihm behandelten Irrtum $\$ 16$ Abs. 1 Satz 1 StGB als Ballast abgeworfen hat, insbesondere mit dem Argument, die Lehre von den negativen Tatbestandsmerkmalen sei axiologisch verfehlt (S. 159 ff; $\mathbb{} 16$ Abs. 1 Satz 1 StGB als „grobschlächtig“, S. 299), ist der Weg frei, nach $\$ 17$ StGB beim „unverständigen Versuch der Rechtfertigung “ Vorsatzschuld auszumachen und ansonsten nach dem „Maß der in ... (dem jeweiligen; G.J.) Irrtum ausgedrückten Rechtsuntreue bzw. -feindschaft“ zu entscheiden. Dieses Maß soll „möglichst proportional in die Zurechnung der Rechtsfolgen " umgesetzt werden (S. 294/320 ff.). - Das größere Problem der Regelungen des Tatvorsatzes ist umgangen worden.

84 Zumal nicht groß im Blick auf die erfolgsqualifizierten Delikte, die einiges vom dolus indirectus auffangen; zum „Ärgernis“ dieser Delikte Jakobs, Gleichgültigkeit (Fn. 80), S. 596; LK-Vogel (Fn. 7), vor \15 Rn. 35 f.; eher zufrieden mit dem Stand de lege lata Gaede, Vorsatz (Fn. 83), S. $260 \mathrm{ff} . / 265 \mathrm{ff}$.

85 U. Kindhäuser, Gleichgültigkeit als Vorsatz, in: Arnold u. a. (Hrsg.), Festschrift für Albin Eser, München 2005, S. 345 (357).

86 Kindhäuser, Gleichgültigkeit (Fn. 85), S. 354. - Beiläufig, mit hypothetischer Rechtstreue darf die Vermeidbarkeit im Bereich des Tatbestands nicht gebildet werden, sondern nur mit (wertungslos vorausgesetztem) anderen Wollen; siehe Jakobs, Strafrecht AT (Fn. 10), Abschn. 6 Rn. 26 f.

87 Dieser Anlass ist objektiv, nicht aber (wie E. Horn, Verbotsirrtum und Vorwerfbarkeit. Eine systematische Grundanalyse der Schuldtheorie, Berlin 1969, S. $87 \mathrm{ff} . / 99 \mathrm{ff}$. und passim postuliert) psychologisierend zu bilden. Zur Problematik der objektiven Bestimmung eingehend Velten, Normkenntnis (Fn. 27), S. $119 \mathrm{ff} . / 313 \mathrm{ff}$. 
bestands ist alles, was einen an Folgenvermeidung interessierten Handelnden zu einer Verhaltensänderung brächte, und für einen solchen Interessierten wäre eine bis $d a$ to praktizierte Gleichgültigkeit allemal ein Anlass zum Innehalten. Das Argument schmilzt also zu der Behauptung zusammen, Vorsatzstrafe setze eine psychisch realisierte Erfolgswahrscheinlichkeit voraus, und das Problem der Genese eines Nichtwissens aus einem Wertungsfehler wird nicht berührt.

Weiterhin wird gegen die Behandlung des dolus indirectus als dolus eingewendet, eine Bestrafung des Gleichgültigen als Vorsatztäter laufe auf eine Strafbarkeit hypothetischer Taten hinaus; denn „in der Sache“ solle bestraft werden, wer die Tat auch bei gegebenem präsenten Wissen begangen hätte. ${ }^{88}$ Jedoch wird nicht ein hypothetisches Verhalten geahndet, sondernd das realiter über Gleichgültigkeit vermittelte. ${ }^{89}$ Doloses Verhalten muss nicht stets ein solches mit präsentem Wissen von der Tatbestandsverwirklichung sein, sondern ist jedes Verhalten, das durch einen Wertungsfehler vermittelt wird. ${ }^{90}$ Deshalb besteht auch kein Hindernis, die mit dolus indirectus herbeigeführten Folgen im strafrechtsfunktionalen Sinn als gewollte Folgen zu bezeichnen (siehe oben A.II.4.). Zwar ist die Möglichkeit des Eintritts der Folgen nicht psychisch präsent, aber das hindert nicht daran, sie zum Wollensinhalt zu rechnen; denn die Folgen liegen in einem Bereich, den der Täter durch seine Gleichgültigkeit ausklammert, und dieses Ausklammern ist ein Urteil über die gesamten in diesem Bereich belegenen orientierenden Faktoren: Was sich auch immer abzeichnen mag, es wird vorab als nicht verhaltensbestimmend ausgeschieden.

\section{Dolus indirectus und geltendes Recht. Zugleich Ergebnis zu „Präsenz des Wissens“}

Was lässt sich im geltenden Recht mit dieser Erkenntnis anfangen? Es sind drei Antworten möglich: (1) Der dolus indirectus wird in $\$ 16$ Abs. 1 StGB (Überschrift!) als „Irrtum“ bezeichnet (nicht etwa: als solcher begriffen), und das bindet. (2) Da der

88 J. Vogel, Normativierung und Objektivierung des Vorsatzes?, GA 2006, S. 386 (389); LK-Vogel (Fn. 7), vor $\$ 15 \mathrm{Rn} .70$.

89 Es kann also keine Rede davon sein, auch unvermeidbares Verhalten müsse bei Gleichgültigkeit zur Vorsatzstrafe (wegen eines dolus indirectus) führen; so aber J. Vogel, Normativierung (Fn. 88), S. 389 Fn. 17; LK-Vogel (Fn. 7), vor $\$ 15$ Rn. 70 Fn. 146. Im Fall der Unvermeidbarkeit vermittelt die Gleichgültigkeit keinen Willensfehler. - Es geht im Ergebnis also um wirkliche, nicht etwa bypothetische Zusammenhänge. - Köhler, Strafrecht AT (Fn. 8), S. 369 mit Fn. 18 vermisst den „Selbsteinbezug “ des Subjekts: An dessen Stelle tritt die Selbstausklammerung.

90 Vogel, Normativierung (Fn. 88), S. 389 Fn. 18; LK-Vogel (Fn. 7), vor $\$ 15$ Rn. 70 mit Fn. 147 (siehe auch Gaede, Vorsatz (Fn.83), S. 270 Fn. 156) legt Wert darauf, den dolus indirectus durch einen Verweis auf Mezger zu desavouieren, der die Bestrafung von „Rechtsblindheit" und „Tatsachenblindheit“ mit Blick auf die „gesunde Volksanschauung“ postuliert hat (E. Mezger, Rechtsirrtum und Rechtsblindheit, in: P. Bockelmann u. a. (Hrsg.), Probleme der Strafrechtserneuerung (Festschrift für Eduard Kohlrausch), Berlin 1944, S. 180 (184); zur „Tatsachenblindheit“ zurückgenommen: ders., Moderne Wege der Strafrechtsdogmatik, Berlin 1950, S. 45 f.), und diese Anschauung ist bekanntlich der Platzhalter für nationalsozialistisches Denken. Aber aus dieser materialen Entgleisung (formal dürfte der ehemalige Neukantianer Mezger auch 1944 noch sehr wohl das Problem des Zusammenhangs von Wertung und Tatsache kennen) folgt kein Gebot, den Befund, der rechtlich bewertet werden muss, allein im präsenten Willensinhalt zu suchen und ja nicht in der Genese des Wollens aus einem Wertungsfehler. 
dolus indirectus nun einmal kein Irrtum ist, steht er rechtlich für Kenntnis. Diese beiden Versionen vergewaltigen die Sprache, und deshalb spricht einiges dafür, (3) den dolus indirectus als eine der im AT häufigen, nicht ausdrücklich geregelten Rechtsfiguren zu behandeln, dies mit der Folge einer Stellung nahe beim Vorsatz und weit entfernt vom vermeidbaren Tatbestandsirrtum. ${ }^{91} \mathrm{Wahrscheinlich}$ wird den Vertretern der seit Feuerbach psychologisierenden Vorsatzlehre ${ }^{92}$ dieser Sprung über den langen Schatten des Stammvaters der Rechtswissenschaft nicht gelingen. ${ }^{93}$ Unterbleibt der Sprung, so kann die oben aufgedeckte, zwingende Verbindung von Tatbestandsvorsatz und Unrechtseinsicht nicht offen thematisiert werden: Der nach \$17 Satz 2 StGB nicht zur Strafmilderung führende so genannte Irrtum erzwänge ansonsten als Unkenntnis nach $\mathbb{} 16$ Abs. 1 den Übergang von einer Vorsatzstrafe zur Fahrlässigkeitsstrafe. Soll das - ohne Anerkennung eines dolus indirectus - vermieden werden, so bleibt nur eine Dogmatik des Als-Ob: Die Trennbarkeit muss fingiert werden.

\section{Jedes Wissen?}

\section{Sonderwissen}

Präsentes Wissen von der Tatbestandsverwirklichung ist, wie der dolus indirectus lehrt, für doloses Verhalten nicht notwendig; es bleibt zu prüfen, ob es stets hinreicht. Die Antwort liegt auf der Hand: Ohne Bezug auf einen objektiven Tatbestand reicht Wissen nicht hin, wie das Erfordernis einer Garantenstellung beim Unterlas-

91 Allerdings wird behauptet, eine Normativierung des Vorsatzbegriffes dürfe das Wissen als psychisches Faktum nicht bereichsweise eskamotieren (Puppe, Vorstellungsinhalt (Fn. 35), S. 36 f; Gaede, Vorsatz (Fn. 83), S. 266 f; wohl auch Schünemann, Vorsatzbegriff (Fn. 35) S. 373; dieses Verständnis dürfte sehr weit überwiegender Ansicht entsprechen und meistens als geradezu selbstverständlich vorausgesetzt werden.). Das ist nicht richtig; denn das Recht entscheidet selbst, woran es anknüpft, und die Meinung, dies dürfe beim Vorsatz immer nur ein präsentes Wissen und keinesfalls ein das aktuelle Verhalten gestaltender Wertungsfehler sein, zeugt von einem falschen Verständnis des Verhältnisses von Recht und Tatsachen. $\mathrm{Ob}$ an Wissen oder an Gleichgültigkeit oder an anderes anzuknüpfen ist, gibt nicht irgendeine Wirklichkeit vor, sondern die Wirklichkeit des gesellschaftlichen Daseins, die analytisch zu erschließen ist, und das lässt sich durch eine Abwertung der am Bewertungsfehler orientierten Lehre als täter- oder gesinnungsstrafrechtlich orientiert (Gaede, Vorsatz (Fn. 83), S. 271; Walter, Kern (Fn. 51), S. 255) nicht leisten (zur „Gesinnung“ siehe den recht ungenierten Sprachgebrauch bei Schünemann, Vorsatzbegriff (Fn. 35), S. 372 ff.): Wenn der durch Wissen vermittelte Willensfehler, also der unbestrittene Fall des Vorsatzes, keinem Täter- oder Gesinnungsstrafrecht zugehört, - wieso dann der durch Gleichgültigkeit vermittelte? Und schon gar nicht stempelt eine Lehre diejenigen Täter zu „Unpersonen“ ab, die sie als Vernünftige beurteilt und nicht nach dem, was Individuen bei der Tat gerade durch den Kopf gehen mag; denn diese Täter werden nicht als Gefahrenquellen exkludiert, sondern als kompetent dem Recht Widersprechende anerkannt (weit entfernt von der Sache deshalb Gaede, Vorsatz (Fn. 83), S. 272).

92 Feuerbach, wie Fn. 70.

93 Für alle nicht oder allenfalls de lege ferenda Sprungbereiten bietet Art. 103 Abs. 2 eine bequeme Ausrede. - Wenn de lege ferenda für Einzelfälle Korrekturen (meist unter dem Namen einer erweiterten Bestrafung fahrlässigen Verhaltens) vorgeschlagen werden (Walter, Kern (Fn. 51), S. 409; Gaede, Vorsatz (Fn. 83), S. 277 ff; T. Hörnle, Das Irren über das Einverständnis des Opfers einer sexuellen Nötigung, ZStW 112 (2000), S. 356 (361 ff./367 ff./371 ff.)), so fehlt dem ohne Offenlegung des allgemeinen Prinzips eine leitende Theorie, so wie die erfolgsqualifizierten Delikte mangels einer solchen Theorie in der Flickschusterei stecken geblieben sind. Nota bene, bei der Umsetzung einer Theorie mögen Praktikabilitätserwägungen eine Rolle spielen, aber bei ihrer Entwicklung sind sie fehl am Platz. 
sungsdelikt belegt; das Wissen eines Nicht-Garanten vom Rettungsweg macht ihn nicht haftbar, genauer, sein Wissen ist kein tatbestandliches, da der Bezug auf eine Garantenstellung fehlt. Unter der Bezeichnung als Sonderwissen wird diskutiert, ob das nicht in bestimmten Fällen anders zu sehen sein soll. Diese Fälle sind zwar für die Praxis ohne ersichtliche Bedeutung, aber bestens geeignet, den Umfang einer Theorie zum Vorsatz sauber darzustellen.

Um Sonderwissen handelt es sich nicht bereits dann, wenn jemandem zufällig etwas „vor die Augen getreten“ ist, anderen hingegen nicht. Beispielhaft, wer zufällig sieht, dass auf einem Stuhl die Brille eines Gastes liegt, darf nicht einen anderen Gast auffordern, sich dort hinzusetzen. In solchen Fällen handelt es sich um erweitertes Alltagswissen. ${ }^{94}$ Demgegenüber ist Sonderwissen dadurch ausgezeichnet, dass es nur kraft einer Befähigung zustande kommt, um die es im gegebenen Zusammenhang nicht geht: Das Wissen gehört weder zu der Rolle, die zu spielen ist, noch handelt es sich um Alltagswissen, wie es jeder in dürftigem, durchschnittlichem oder erweitertem Umfang mitbringt, mit anderen Worten, es gehört nicht zu dem Bereich, in dem der Agierende Schadlosigkeit zu garantieren ${ }^{95}$ hat. Es gibt keinen zwingenden $\mathrm{Zu}$ sammenhang von Wissen und Pflichtenumfang; Sonderwissen ist demgemäß ein Wissen ohne zugehörigen objektiven Tatbestand, wie beim Wissen des unterlassenden Nicht-Garanten.

Die überwiegende Lehre berücksichtigt freilich bei der Bestimmung der Zuständigkeit (im Rahmen der objektiven Zurechnung) neben dem rollengemäßen Wissen und dem Alltagswissen auch dieses besondere Wissen des Agierenden. Jedes Wissen soll also eine Garantie begründen können. ${ }^{96}$ Ein Beispiel: ${ }^{97}$ Ein fortgeschrittener Biologiestudent jobbt in den Semesterferien als Kellner und erkennt aufgrund seines im Studium erworbenen Wissens in einem zu servierenden Salat eine sehr giftige Pflanze, die einer Sorte Petersilie, die sich auch im Salat befindet, täuschend ähnlich sieht; er

94 Für dieses Alltagswissen (nicht aber für das Sonderwissen) gilt die Feststellung von Köhler, Strafrecht AT (Fn. 8), S. 184, es handele sich um „erweitertes Allgemeinwissen“.

95 Auch beim Begehungsdelikt muss der Täter Garant der Schadensvermeidung sein; dazu Jakobs, wie Fn. 67.

96 Die übliche Begründung lautet, es gehe um einen „besonnene(n) Angehörige(n) des maßgeblichen Verkehrskreises in der Lage des Täters" (R. Kaminski, Der objektive Maßstab im Tatbestand des Fahrlässigkeitsdelikts, Berlin 1992, S. 86 ff./87; Hervorhebung nicht original). - Eingehende Nachweise bei L. Greco, Das Subjektive an der objektiven Zurechnung: Zum „Problem“ des Sonderwissens, ZStW 117 (2005), S. 519 (519f. Fn. 6); dort S. 521 ff./534 ff. auch Stellungnahmen zum Verhältnis von Sonderwissen und objektiver Zurechnung. U. Murmann, Zur Berücksichtigung besonderer Kenntnisse, Fähigkeiten und Absichten bei der Verhaltensnormkonturierung, in: Dannecker (Fn. 69), S. 134 ff., will auf den Zweckzusammenhang abstellen und etwa bei der „Einhaltung der Regeln des Straßenverkehrs“ danach entscheiden, ob das Fahrzeug als „Fortbewegungsmittel“ oder „zum Herbeiführen von Unfällen “ (auch: zum Waffenhandel oder zur Ausbeutung der Prostitution?) eingesetzt wird (S. 135 f.). Es müsste freilich begründet werden, dass auf die Innerlichkeit des Agierenden überhaupt zugegriffen werden darf, solange das äußere Verhalten korrekt ist.

97 Nach G. Jakobs, Tätervorstellung und objektive Zurechnung, in: Dornseifer u. a. (Hrsg.), GS Armin Kaufmann (Fn. 14), S. 271 (273); ders,. Strafrecht AT (Fn. 10), Abschn. 7 Rn. 49 f.; ders, Norm (Fn. 60), S. 98. 
serviert ungerührt. Die weit überwiegende Lehre will, wenn der Gast an dem Gift stirbt, wegen einer Tötung durch Tun bestrafen. ${ }^{98}$ Lässt aber der Student als Kellner seinen Blick auf der Pflanze ruhen, ohne sie zu identifizieren, was ihm freilich sehr leicht möglich wäre, soll er straffrei ausgehen (dazu im nächsten Absatz). Also soll die Berücksichtigung von Wissen auch dann zu garantieren sein, wenn zu seiner Aktivierung keine Pflicht besteht? Die bejahende Antwort der üblichen Lehre ${ }^{99}$ zeugt von dem schon mehrfach aufgewiesenen Psychologismus (das Faktum des Bewusstseins regiert) und ist deshalb per se keine Aussage über die rechtliche Struktur. - Ein weiteres Beispiel: ${ }^{100}$ Ein Kraftfahrzeugingenieur, Spezialist für Bremstechnik, fährt einen Leihwagen und schließt aus nur wenigen Spezialisten erkennbaren Indizien auf einen demnächst (aber nicht schon bei seiner Fahrt) eintretenden Bremsdefekt; er gibt das Fahrzeug zurück, ohne auf die Gefahr hinzuweisen. Der nächste Mieter des Fahrzeugs verunglückt. Sollte der Ingenieur als Täter einer vorsätzlichen Schadensherbeiführung einstehen müssen? ${ }^{101}$

Das Sonderwissen bezieht sich auf einen Gegenstand, um den der Täter sich nicht kümmern muss, juristisch exakter formuliert, der außerhalb der von ihm als Garant zu verwaltenden Risiken liegt. ${ }^{102}$ Das wird insbesondere dann deutlich, wenn der Bereich des - nun einmal sinnenfällig imponierenden - faktischen Wissens verlassen

98 Murmann, Berücksichtigung (Fn. 96), S. 126, 132, der freilich den Kellner zu einer Art Mit-Garanten für die Essensqualität stilisiert; M. Sacher, Sonderwissen und Sonderfähigkeiten in der Lehre vom Straftatbestand, Berlin 2006, S. 103/287 und passim mit Nachweisen; dies., Systemtheorie und Strafrecht, ZStW 118 (2006), S. 575 (617). Als Argumente fungieren bei Sacher: „Kapitaldelikt“, „finale Steuerung des Geschehens“, „Schutzlosigkeit des Opfers“, „Ausschluss der Zurechnung ... nicht akzeptabel“ (alle Zitate: Systemtheorie, S. 617). Die erste und die letzte Aussage sind bloße Behauptungen, die beiden mittleren geben Fakten wieder; die Ebene der rechtlichen Struktur der Gesellschaft wird also nicht erreicht.

99 Eingehend Greco, wie Fn. 96. - Seine Begründung dürfte falsch sein: Auch wenn es keine Pflicht zur Beschaffung von Sonderwissen gebe, müsse es eingesetzt werden, wie auch ein Hund der erlaubterweise angeschafft wurde, überwacht werden müsse (Fn. 96), S. 543. Aber Sonderwissen begründet keine Gefahr, und es geht auch weniger um den Erwerb dieses Wissens, als um seine Aktivierung.

100 Nach Jakobs, Strafrecht AT (Fn. 10), Abschn. 7 Rn. 50.

101 Das wegen der Bindung an psychische Fakten rechtlich notwendig Aleatorische der Entscheidung nach der üblichen Ansicht wird bei Sacher deutlich, wenn sie bei „Bedarf“ vom Tun zum Unterlassen wechselt, um dann wegen Fehlens einer Garantenstellung Zurechnung verneinen zu können: Im Fall des Ingenieurs soll es sich, im Gegensatz zum Kellnerfall, „letztendlich“ um ein Unterlassen handeln (Sonderwissen, Fn. 98), S. 103 f.; ebenso Murmann, Berücksichtigung (Fn. 96), S. 132 f. Nun mögen die Übergabe eines Salates und diejenige eines Automobils, jeweils durch eine Sequenz von Körperbewegungen, manche phänotypischen Unterschiede aufweisen, aber beides ist - sieht man von offenbaren Verlegenheitslösungen $\mathrm{ab}$ - nach üblicher Klassifikation ein Tun. - Zur Notwendigkeit einer Garantenstellung auch beim Tun siehe Jakobs, wie Fn. 67.

102 Was zu verwalten ist und was nicht, richtet sich (auch) nach dem Kontext, in dem der Täter sich darstellt. Beispielhaft, wer in einer Kleinstadt die Rolle eines für jeden verfügbaren Arztes spielt, kann aus dieser Rolle auch dann nicht schlüpfen, wenn er zufällig bei einem Unfall anwesend ist. Unter anonymisierenden Verhältnissen stellt sich das freilich anders dar! Faustformel: Alltäglich praktizierte Fähigkeiten sind stets einzusetzen; Sonderwissen kann es nur bei trennbaren Rollen geben. Die insoweit erforderlichen Analysen der normativen Struktur - die hiesigen Ausführungen können nur eine Skizze bieten, keine genaue Karte - werden durch den von der üblichen Lehre immer wieder bemühten Naturalismus Wissen/Unkenntnis zurückgedrängt: Gegen bequeme alltagsmoralische Evidenzen haben es rechtliche Argumente manchmal schwer! 
und nach der Fahrlässigkeit gefragt wird: Gibt es, traditionell gesprochen, im Bereich des Sonderwissens Sorgfaltspflichten? Die Frage wird weit überwiegend verneint und zwar im Ergebnis mit Recht; man muss ein Verhalten nur dann vermeiden, wenn die als Garant zu verwaltenden Risiken nicht sicher ausgeschlossen werden können. Angewandt auf die oben genannten Beispiele heißt das nicht nur, der Kellner müsse sich den Salat nicht näher ansehen oder der Ingenieur müsse keinen Blick auf die Bremsanlage werfen, sondern auch nach der Identifizierung der Pflanze oder nachdem eine schwache Schraube an stark beanspruchter Stelle gesehen wurde, dürfe dem Täter der Fortgang gleichgültig bleiben; ${ }^{103}$ er hat eben nicht zu garantieren, dass solche Risiken nicht auftreten und darf deshalb auch das, was er festgestellt hat, sofort wieder vergessen. Was dem Täter, wenn er aufmerksam ist, vor Augen steht, ist ein kommendes Unglück, nicht aber ein von ihm organisierter Schadensverlauf: Er weiß, dass es bei einem normalen Fortgang des Geschehens zu einem Unglück kommen wird, freilich zu einem solchen, dem er durch ein außergewöhnliches (wenn auch sehr leicht zu vollziehendes) Verhalten zuvorkommen könnte, wobei es in nicht wenigen Fällen - wie in allen beispielhaft angeführten - nicht genügen würde, eine bestimmte Handlung zu unterlassen (den Salat nicht zu servieren; das Fahrzeug nicht zurückzugeben), vielmehr die aktive Vorsorge hinzutreten müsste, dass nicht andere Personen die ausbleibende Handlung arglos ersetzen. Bloßes Unterlassen bringt allenfalls eine Zufallsrettung.

Gewiss ist es ein Skandal, wenn ein Täter ein sehr leicht zu vermeidendes großes Unglück nicht vermeidet, aber dieser Skandal hängt nicht davon ab, wie sich der Täter zuvor verhalten hat, sondern folgt aus der starken moralischen und schwachen rechtlichen Erwartung, er werde, da er als einziger die Lage überblickt, bei größter Not, aber auch nur dann, für die Sicherheit des Gefährdeten sorgen. Diese Sorge für

103 Wenig hilfreich Sacher, Sonderwissen (Fn. 98), S. 262 f.: „Das Sonderwissen muss ... auch bei Fahrlässigkeitsdelikten zu Lasten des Täters gehen, wenn das Wissen über risikosteigernde Faktoren so aktualisiert ist, dass eine Sorgfaltspflicht zur Vermeidung rechtsverletzender Folgen entsteht." Gemeint ist wohl eine Wissenslage mit Elementen, die sich bei sorgfältiger Sichtung zu einem Risikosyndrom verbinden lassen. Aber woher soll die Pflicht zur Sichtung rollenübersteigender Wissenselemente (oder zur vorsorglichen Verhaltensänderung) kommen? Bei Sacher degeneriert die normative Struktur der Gesellschaft zum Derivat psychischer Vorgänge, nicht anders als bei allen Autoren, die beim Sonderwissen aus dem Können auf ein Sollen schließen; so verfährt auch Kindhäuser, Der subjektive Tatbestand im Verbrechensaufbau. Zugleich eine Kritik der Lehre von der objektiven Zurechnung, GA 2007, S. 447 (460 ff./462), der zutreffend darlegt, der mit einem Sonderwissen ausgestattete Täter sei fähig, den Schadensverlauf zu vermeiden, aber nicht begründet, warum er als $G a$ rant auch vermeiden soll (so zutreffend kritisch $H$. Kawaguchi, Dolus indirectus im Spiegel der japanischen Rechtsprechung, in: Pawlik et al. (Hrsg.), FS Jakobs (Fn. 68), S. 259 (262). Die Argumentation ist einer ökonomischen Ausrichtung des Rechts näher als der Analyse von Rechten und Pflichten. - Siehe auch G. Arzt, Dolus eventualis und Verzicht, in: K. Rogall et al. (Hrsg.) FS Rudolphi (Fn. 64), S. 3 (12): Zur Rolle eines Apothekers, der ein geläufiges Mittel aushändigt, das allerdings, wie er weiß, missbraucht werden wird, gehöre keine Mordteilnahme. Diese müsste freilich als Pflichtverletzung und nicht durch den bloßen Hinweis auf den Kausalverlauf begründet werden. Zum Verhältnis von Vorsatz und normativer Gesellschaftsstruktur siehe auch L. C. Rey-Sanfiz, Die Begriffsbestimmung des Versuchs und ihre Auswirkung auf den Versuchsbeginn, Berlin 2006, S. $260 \mathrm{ff}$. 
Sicherheit ist freilich ohne den Bestand einer besonderen Verbindung keine Aufgabe einer ihren eigenen Organisationkreis verwaltenden Person im Recht, vielmehr eine solche des Staates, und demgemäß gibt es keine Garantenpflicht der Person zu einer entsprechenden Besorgung, sondern allenfalls eine eigentümliche Pflicht des Bürgers, der den Staat, dessen in erster Linie berufene Amtswalter mangels Kenntnis nichts ausrichten können, zu vertreten hat. Die öffentliche Person, eben der Bürger, wird in ein - kleines und nur temporäres - Amt berufen: in einem Einzelfall für Sicherheit zu sorgen, wobei die Verletzung dieser Pflicht positivrechtlich durch \323c StGB erfasst wird. ${ }^{104}$

Damit liegt auch die Antwort auf die Frage nahe, weshalb zur Verwirklichung des Tatbestands der „unterlassenen“ Hilfeleistung positive Kenntnis der Schadenslage erforderlich ist, aber keine Sorgfalt aufgebracht werden muss, sie zu erlangen, - eine Frage, die von der üblichen Lehre kaum gestellt wird: Die Bürger sollen nicht dauernd die Grenzen der Wirksamkeit des Staates belauern müssen, sondern nur dann in ihre Tätigkeit als Substituten eintreten, wenn sie augenfällig dazu berufen werden, wenn ihnen also das Amt in diesem Sinne „angetragen“ wird. ${ }^{105}$

\section{Besonderheiten}

\section{a. Garanten}

Freilich mag eine Person in einer Verbindung mit einer anderen stehen, die sie verpflichtet, nicht nur das von jedermann zu Erbringende zu leisten, sondern - etwa alles Erforderliche. So verhält es sich im Bereich der negativen Pflichten ${ }^{106}$ beispielsweise, wenn nach einem rechtswidrigen Angriff der Angreifer wegen seiner Pflichten aus Ingerenz einen Schaden abzuwenden hat: Wie bei der Notwehr gegen einen solchen Angriff alles Erforderliche unternommen werden darf, der Angreifer also nicht nur bereichsweise, sondern umfassend opferpflichtig ist, so kennt auch die Verlängerung dieser Pflicht in der Ingerenz keine Bereichsgrenzen. - Damit soll nicht behauptet werden, bei schlechthin allen Garantenstellungen sei ohne Blick auf Standards subjektiv Optimales zu leisten; so ist etwa bei einer Übernahme ${ }^{107}$ das Übernommene aufzubringen, nicht mehr. Sind aber nun einmal besondere Fähigkeiten

104 Siehe dazu das Konzept des Hilfsleistungs- „Gebots“ bei M. Pawlik, Unterlassene Hilfeleistung: Zuständigkeitsbegründung und systematische Struktur, GA 1995, S. 360 (365); auch ders., Der rechtfertigende Notstand. Zugleich ein Beitrag zum Problem strafrechtlicher Solidaritätspflichten, Berlin 2002, S. 179 ff./182 ff. und passim. - M. Sancinetti, Subjektive Unrechtsbegründung und Rücktritt vom Versuch, Köln et al. 1995, S. 245 f., Fn. 28, stellt die nahe liegende Frage, wieso bei $\$ 323$ c StGB ein Sonderwissen zu berücksichtigen sein soll. Eine solche Berücksichtigung ist nicht selbstverständlich, sondern überhaupt nur möglich, wenn gilt: Größte Not sprengt alle Rollen. Versteht man die der Vorschrift zugrunde liegende Norm hingegen technokratisch (als Mittel zur Maximierung von Wohl), mag ein Sonderwissen zu den vorbehaltenen (nicht in das Rechtsverhältnis eingebrachten) Fähigkeiten gehören.

105 Verkannt bei Sacher, Sonderwissen (Fn. 98), S. 104 f.

106 Es geht um die Pflichten, nicht zu verletzen; siehe Jakobs, Strafrecht AT (Fn. 10), Abschn. 7 Rn. 56 ff.

107 Jakobs, Strafrecht AT (Fn. 10), Abschn. 29 Rn. 46 ff.; zum sonstigen Maß des zu Leistenden $\mathbb{2 9}$ Rn. 14. 
einzusetzen, so geht es, was Kenntnisse angeht, nicht nur um präsentes Wissen, sondern auch um erreichbares.

Eine besondere Verbindung - nunmehr im Bereich positiver Pflichten ${ }^{108}$ - besteht auch, soweit eine Person verpflichtet ist, mit einer anderen eine gemeinsame Welt zu bilden, wie insbesondere Eltern gegenüber ihren Kindern. So wie sich die Eltern in ihren anderen Rollen - als Angehörige ärztlicher, kaufmännischer und anderer Berufe, als Sportler oder Jäger - nicht dergestalt von ihren Kindern distanzieren können, dass deren Hilfsbedürftigkeit sie nichts mehr anginge, so können sie ihnen auch nicht ihr Wissen vorenthalten, das sie in diesen anderen Rollen erworben haben. Beispielhaft, die Mutter, die aufgrund ihres früheren Medizinstudiums bestimmte Krankheitssymptome erkennt, hat ihr Wissen umzusetzen, also zweckvolle Maßnahmen zur Heilung einzuleiten. - Weitere besondere Verbindungen, die eine Rollentrennung ganz oder doch partiell ausschließen, dürften sich finden lassen; insbesondere sind Sonderfähigkeiten einzubringen, wenn der Täter die Rolle eines Sonderbegabten spielt.

\section{b. Eingebrachtes Sonderwissen}

Durchbricht der Täter seinerseits eine an sich bestehende Rollentrennung, bringt er also sein Wissen aus einer Rolle in eine andere ein, so wandelt sich das Sonderwissen zum schlichten Wissen von einer bevorstehenden Tatbestandsverwirklichung. Stellt also im bereits vielfach herangezogenen Beispiel der Biologiestudent als Kellner den Salat mit der Giftpflanze beiseite, bis er ihn seinem Erbonkel servieren kann, handelt es sich, wenn der Onkel stirbt, um vorsätzliche Tötung. ${ }^{109}$

Insoweit abschließend: Auch ein Wissen von der Gefabrlosigkeit kann eingebracht werden. Als Abwandlung eines Beispiels von Armin Kaufmann ${ }^{110}$ nehme man an, ein Kraftfahrzeughalter sehe einen kräftigen Riss im Lenkgehäuse seines Fahrzeugs, den nahezu alle aufmerksamen Halter als höchst gefährlich einstufen würden; er selbst ist aber ein mit solchen Aggregaten erfahrener Ingenieur und weiß, dass der Riss nur durch abdeckende, nicht aber durch Stabilität garantierende Teile des Gehäuses verläuft, und wegen dieses Wissens (Er bringt sein Ingenieurswissen in seine Rolle als Fahrzeughalter ein!111) fährt er bedenkenlos. Seine Fahrt ist mangels Vor-

108 Grundlegend Sánchez-Vera, Pflichtdelikt (Fn. 66), S. 51 ff./126ff.; ferner Jakobs, Strafrecht AT (Fn. 10), Abschn. 7 Rn. 70 f., $\$ 29$ Rn. 57 ff.

109 Dazu Jakobs, Strafrecht AT (Fn. 10), Abschn. 7 Rn. 49 ff. - Greco, Zurechnung (Fn. 96), S. 544, wendet ein, der Kellner könnte die Erlaubnis gehabt haben, das Gericht einer Person seiner Wabl zu servieren. Aber dann darf er die Wahl nicht nach seiner Kenntnis richten, oder er bringt die Kenntnis ein und haftet!

110 Armin Kaufmann, Das fahrlässige Delikt, Zeitschrift für Rechtsvergleichung, 1964, S. 41 (49), dort wird der Fall freilich ohne Sonderwissen gebildet.

111 Das verkennen I. Puppe, Der Aufbau des Verbrechens, in: Dannecker et al. (Hrsg.) FS Otto (Fn. 69), S. 389 (396 f.); Greco, Zurechnung (Fn. 96), S. 544/547; zu einem anderen Beispiel auch verkannt von Kindhäuser, Tatbestand (Fn. 103), S. 460: die Schadlosigkeit ist dann Element des Grundes, aus dem die Handlung vollzogen wird. 
satzes kein Versuch der Delikte, die sich bei einem wirklichen Defekt der Lenkung einstellen könnten, und ist bei dem gegebenem Vorrang seiner Sicht der Dinge auch kein - per se ohnehin straffreier - fahrlässiger Versuch.

\section{Ergebnis zu „Jedes Wissen?“}

Wissen von der Gefährlichkeit eigenen Verhaltens oder von der Möglichkeit, einen bestehenden Schadensverlauf durch eigenes Verhalten zu inhibieren, bezieht sich nur in demjenigen Umfang auf die Pflicht, die einem Verletzungsdelikt zugrunde liegt, in dem der Täter Schadlosigkeit zu garantieren hat, wobei diese Garantie nicht anders zu bestimmen ist, als es bei einem Unterlassungsdelikt geläufig geschieht. Die für Verletzungsdelikte, die durch Unterlassen verwirklicht werden, nahezu unbestrittene Regelung gilt auch bei aktivem Tun, soweit das Wissen kein Alltagswissen ist und auch nicht zu der Rolle gehört, in der sich der Agierende bewegt. Ohne die Garantie - ein Element des objektiven Tatbestands - ist ein Wissen allenfalls (bei ansonsten drohenden Katastrophen) vom Bürger als Substitut des Staates einzusetzen $(\mathbb{S} 323 \mathrm{c}$ StGB). 